\title{
Synthesis of [1]benzothieno[3,2-b][1]benzothiophene (BTBT) and its higher homologs through palladium-catalyzed intramolecular decarboxylative arylation
}

\author{
Sojiro Minami $^{\mathrm{a}}$, Koji Hirano ${ }^{\mathrm{a}}$, Tetsuya Satoh $^{\mathrm{a}, \mathrm{b}}$ and Masahiro Miura ${ }^{\mathrm{a}, *}$ \\ ${ }^{a}$ Department of Applied Chemistry, Faculty of Engineering, Osaka University, Suita, Osaka 565-0871, Japan \\ ${ }^{b} J S T$, ACT-C, 4-1-8 Honcho, Kawaguchi, Saitama 332-0012, Japan \\ *Corresponding author: Tel. +81-6879-7360; fax: +81-6-6879-7362. E-mail address: miura@ chem.eng.osaka-u.ac.jp (M. Miura)
}

\section{ARTICLE INFO}

Article history:

Received

Received in revised form

Accepted

Available online

Keywords:

Arylation

BTBT

Decarboxylation

Palladium

Thienoacene

\section{ABSTRACT}

We report herein an effective method for the construction of 4- and 7-ring benzo-fused thieno[3,2- $b$ ] thiophenes, involving palladium-catalyzed intramolecular decarboxylative arylation as the key step.

2009 Elsevier Ltd. All rights reserved.
Various poly-condensed arenes and heteroarenes are known to work as organic semiconductors. Particularly, thienoacenebased extended $\pi$-systems have attracted much attention, as they are robust and often show high hole mobilities in field effect transistor (FET) devises. ${ }^{1}$ Benzo-fused thieno[3,2- $b$ ]thiophenes such as [1]benzothieno[3,2-b][1]benzothiophene (BTBT) (Figure 1) derivatives are typical promising compounds. Thus, the synthesis of BTBT and its derivatives has been studied extensively in recent years. BTBT itself has been prepared by a number of methods including the reactions of 1,2-bis(2bromophenyl)acetylene with $\mathrm{S}_{8}$ or $\mathrm{Na}_{2} \mathrm{~S},{ }^{2}{ }^{2}$,2'-dichlorostilbene with $\mathrm{Na}_{2} \mathrm{~S}^{3},{ }^{3}$-chlorobenzaldehyde with $\mathrm{NaSH}^{4}{ }^{4}$ and benzyl chloride with $\mathrm{S}_{8} \cdot{ }^{5,6}$ Another effective method leading to BTBT is the iodine-promoted cyclization of 2,2'-bis(methylthio)stilbene, which was also applied to the synthesis of a largely extended thienoacene, i.e. bis[1] benzothieno[2,3- $\left.d ; 2^{\prime}, 3^{\prime}-d^{\prime}\right]$ benzo[1,2$\left.b ; 4,5-b^{\prime}\right]$ dithiophene (BBTBDT) and its naphtho-analog. ${ }^{7}$ BBTBDT was also prepared by a Pummerer-type biscyclization. ${ }^{8}$ Recently, Takimiya and coworkers disclosed an elegant process for the construction of BTBT as well as its higher homologs by the sequential reactions of 2[2-(trimethylsilyl)ethynyl]thioanisole with $\mathrm{PhSCl}$ to form the corresponding 3(phenythio)benzo[ $b]$ thiophene and its 2-bromination followed by palladium-catalyzed intramolecular C-H arylation. ${ }^{9}$ Despite these achievements for constructing the BTBT series of compounds, there still remains a substantial demand for further development of effective and flexible synthetic methods to enhance their utility. We herein describe a new synthetic sequence involving palladium-catalyzed intramolecular decarboxylative arylation as the key step, which enables to construct unknown bis[1]benzothieno[2,3- $\left.d ; 2^{\prime}, 3^{\prime}-d^{\prime}\right]$ benzo[1,2- $\left.b ; 5,6-b^{\prime}\right]$ dithiophene (iso-BBTBDT) skeleton as well as BTBT and BBTBDT and their alkylated derivatives. Decarboxylative arylation for constructing biaryl linkages using widely available arenecarboxylic acids as one of the coupling partners has recently emerged as an effective cross-coupling strategy that complements the relevant conventional methods employing arylmetal reagents. ${ }^{10}$<smiles></smiles>

BTBT

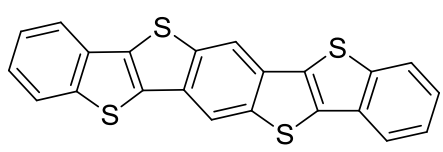

BBTBDT

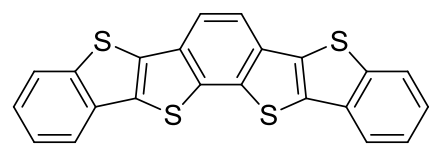

iso-BBTBDT

Figure 1. Structure of BTBT, BBTBDT, and iso-BBTBDT.

We previously reported the synthesis of 3-(arylthio)-2arylbenzo[b]thiophene (4) of pharmaceutical interest (Scheme 1). The method with the readily available methyl 3- 
Table 1. Synthesis of BTBT and $\mathrm{C}_{8}$-BTBT by intramolecular decarboxylative arylation ${ }^{\mathrm{a}}$

\begin{tabular}{cccccc}
\hline Entry & Substrate & Ligand $^{\mathrm{b}}$ & $\mathrm{Base}$ & Time (h) & Product, yield $^{\mathrm{c}}(\%)$ \\
\hline 1 & $\mathbf{3 a}$ & $\mathrm{P}(o-\mathrm{BP}) \mathrm{Cy}_{2}$ & $\mathrm{Cs}_{2} \mathrm{CO}_{3}$ & 2 & $\mathbf{5 a}, 77$ \\
2 & $\mathbf{3 a}$ & $\mathrm{P}(o-\mathrm{BP}) \mathrm{Cy}_{2}$ & $\mathrm{Na}_{2} \mathrm{CO}_{3}$ & 2 & $\mathbf{5 a}, 75$ \\
3 & $\mathbf{3 a}$ & $\mathrm{P}(o-\mathrm{BP}) \mathrm{Cy}_{2}$ & $\mathrm{~K}_{2} \mathrm{CO}_{3}$ & 6 & $\mathbf{5 a}, 71$ \\
5 & $\mathbf{3 a}$ & $\mathrm{P}(o-\mathrm{BP}) \mathrm{Cy}_{2}$ & $\mathrm{CsOAc}$ & 6 & $\mathbf{5 a}, 75$ \\
6 & $\mathbf{3 a}$ & $\mathrm{P}(o-\mathrm{BP}) \mathrm{Cy}_{2}$ & $\mathrm{DABCO}$ & 6 & $\mathbf{5 a}, 15$ \\
7 & $\mathbf{3 a}$ & $\mathrm{PCy}$ & 6 & $\mathbf{5 a}, 90$ \\
8 & $\mathbf{3 a}$ & $\mathrm{P}(o-\mathrm{BP})(t-\mathrm{Bu})_{2}$ & $\mathrm{Cs}_{2} \mathrm{CO}_{3}$ & 2 & $\mathbf{5 a}, 91(80)$ \\
\hline
\end{tabular}

${ }^{\mathrm{a}}$ Conditions: $[3]:\left[\mathrm{Pd}(\mathrm{OAc})_{2}\right]:\left[\mathrm{P}(o-\mathrm{BP})(t-\mathrm{Bu})_{2}\right]:\left[\mathrm{Cs}_{2} \mathrm{CO}_{3}\right]=1: 0.1: 0.2: 2(0.25 \mathrm{mmol}$ of 3 was used $)$, at $160{ }^{\circ} \mathrm{C}$ under $\mathrm{N}_{2}$ in DMAc $(2.1 \mathrm{~mL})$

${ }^{\mathrm{b}} o$-BP $=$ biphenyl-2-yl.

${ }^{\mathrm{c}}$ Determined by GC analysis. Value in parentheses is isolated yield.

${ }^{\mathrm{d}} 0.13 \mathrm{mmol}$ of $\mathbf{3 b}$ was used in $1 \mathrm{~mL}$ of DMAc.

chlorobenzo $[b]$ thiophene-2-carboxylate (1a) as the starting scaffold consists of nucleophilic 3-arylthiolation and palladiumcatalyzed decarboxylative 2 -arylation. ${ }^{11}$ This sequence may allow various 2,3-disubstitution patterns on the benzo[b]thiophene framework. It may be conceived that using 2bromobenzenethiols could afford BTBT and its derivatives via intramolecular decarboxylative arylation. Consequently, we have undertaken to their synthesis based on this strategy.

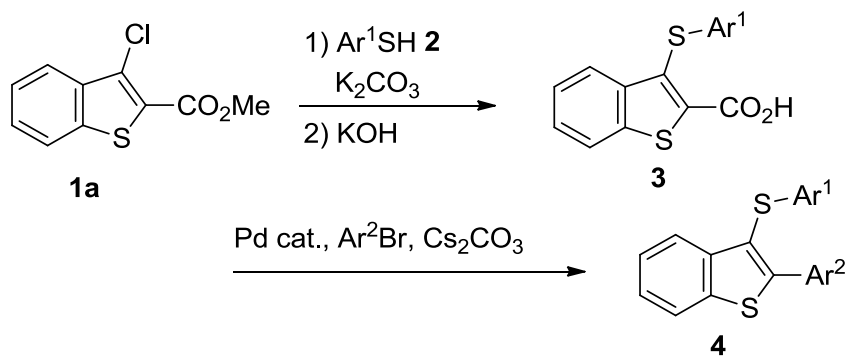

Scheme 1. Preparation of 2-aryl-3-(arylthio)benzo[b]thiophene by nucleophilic arylthiolation and decarboxylative arylation (previous work). ${ }^{11}$

We first examined the synthesis of mother BTBT by using methyl 3-[(trifluoromethyl)sulfonyloxy]-benzo[b]thiophene-2carboxylate (1b) $)^{12}$ as the starting material to test the applicability of the triflate analog of 1a (Scheme 2), since the leaving group is required to construct a higher BTBT analog (vide infra).

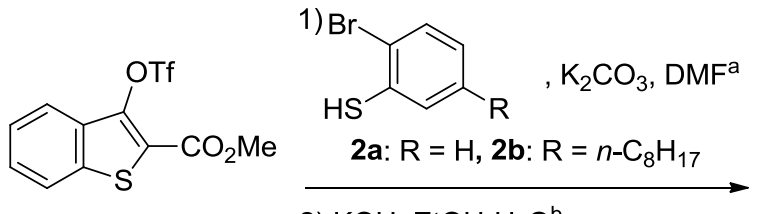

1b: $X=$ OTf

2) $\mathrm{KOH}, \mathrm{EtOH}-\mathrm{H}_{2} \mathrm{O}^{\mathrm{b}}$<smiles>O=C(O)c1sc2ccccc2c1Sc1cc(F)ccc1Br</smiles><smiles>CC(=O)OCCCCCC(C)(C)C</smiles><smiles>[R]c1ccc2c(c1)sc1c3ccccc3sc21</smiles>

5a: $\mathrm{R}=\mathrm{H}(\mathrm{BTBT})$

5b: $\mathrm{R}=n-\mathrm{C}_{8} \mathrm{H}_{17}\left(\mathrm{C}_{8}-\mathrm{BTBT}\right)$
As expected, the reaction of $\mathbf{1 b}$ with 2-bromobenzenethiol (2a) in the presence of potassium carbonate in DMF at $80{ }^{\circ} \mathrm{C}$ gave the corresponding phenylthiolated product in 53\% yield and the subsequent hydrolysis with potassium hydroxide in ethanol/ $\mathrm{H}_{2} \mathrm{O}$ proceeded quantitatively to afford 3-[2(bromophenyl)thio]-2-benzo[b]thiophenecarboxylic acid (3a). Then, the intramolecular decarboxylative arylation of 3a was conducted in the presence of $\mathrm{Pd}(\mathrm{OAc})_{2}$ as catalyst in combination with a number of bases and ligands in DMAc at 160 ${ }^{\circ} \mathrm{C}$ (Table 1). Using $\mathrm{P}(o-\mathrm{BP}) \mathrm{Cy}_{2} \quad(o-\mathrm{BP}=$ biphenyl-2-yl, CyJohnphos) as ligand, various inorganic bases including $\mathrm{Cs}_{2} \mathrm{CO}_{3}, \mathrm{Na}_{2} \mathrm{CO}_{3}, \mathrm{~K}_{2} \mathrm{CO}_{3}$, and $\mathrm{CsOAc}$ could be used to furnish BTBT in $71-77 \%$ yields (determined by GC, Entries 1-4). The relatively strong $\mathrm{Cs}_{2} \mathrm{CO}_{3}$ was found to be superior to the other bases examined. However, a strong organic amine base, $\mathrm{DABCO}$, was far less effective (Entry 5). $\mathrm{PCy}_{3}$ and $\mathrm{P}(o-\mathrm{BP})(t-$ $\mathrm{Bu})_{2}$ (Johnphos) in place of $\mathrm{P}(o-\mathrm{BP}) \mathrm{Cy}_{2}$ afforded higher BTBT yields of $90 \%$ and $91 \%$, respectively (Entries 6 and 7). Thus, BTBT was isolated in $80 \%$ yield in Entry 7. Similarly, the use of 2-bromo-5-octylbenzenethiol $(\mathbf{2} \mathbf{b})^{13}$ in place of $\mathbf{2 a}$ in the starting arylthiolation reaction of $\mathbf{1 b}$ led to 2-octyl-BTBT $\mathbf{5 b}\left(\mathrm{C}_{8}\right.$-BTBT). The isolated yield of $\mathbf{5 b}$ in the decarboxylative arylation step was $72 \%$ (Entry 8). It is worth noting that not only a dialkyl-BTBT, but also a monoalkyl-BTBT has been reported to show a high hole mobility. ${ }^{6}$

We next examined the synthesis of BBTBDT by using $\operatorname{di}(2-$ ethylhexyl) 3,7-bis(trifluoromethanesulfonyloxy)benzo[1,2$\left.b: 4,5-b^{\prime}\right]$ dithiophene-2,6-carboxylate (1c) as the synthetic scaffold (Scheme 3), the preparation of which starting from commercially available 2,5-dibromoterephthalic acid was recently described by us. ${ }^{12}$ The reaction of $1 \mathbf{c}$ with $\mathbf{2 a}$ followed by hydrolysis gave the corresponding diacid $\mathbf{6 a}$ in $42 \%$ yield (by two steps) and the subsequent decarboxylative cyclization afforded BBTBDT in $61 \%$ yield $(18 \%$ after sublimation in vacuo). The physical properties of BBTBDT have been reported. ${ }^{7,8}$ Using $\mathbf{1 c}$ and $\mathbf{2 b}$ could also be obtained 2,9-dioctylBBTBDT ( $2 \mathrm{C}_{8}$-BBTBDT), albeit with a moderate yield. ${ }^{14}$

Scheme 2. Synthesis of BTBT and its alkylated derivative $\left(\mathrm{C}_{8^{-}}\right.$ BTBT). ${ }^{a}$ Conditions: [1 $\left.\mathbf{b}\right]:[\mathbf{2}]:\left[\mathrm{K}_{2} \mathrm{CO}_{3}\right]=1: 1.2: 2(7.1 \mathrm{mmol}$ of $\mathbf{2 a}$ and 0.6 mmol of $2 \mathbf{b}$ were used), $80{ }^{\circ} \mathrm{C}, 8-24 \mathrm{~h}$ in DMF under $\mathrm{N}_{2}$. ${ }^{\mathrm{b}} \mathrm{Conditions:}$ [2]: $[\mathrm{KOH}]=1: 3, \mathrm{EtOH} / \mathrm{H}_{2} \mathrm{O}=10: 1(\mathrm{v} / \mathrm{v}), 100{ }^{\circ} \mathrm{C}, 6-14 \mathrm{~h}$ under $\mathrm{N}_{2}$. Yields of 3a and $\mathbf{3 b}$ were $53 \%$ and $65 \%$, respectively (by two steps). ${ }^{\mathrm{c}}$ See Table 1. 
<smiles>CCOC(=O)c1sc2cc3c(OCC)c(C(=O)OCC)sc3cc2c1O</smiles>

1c $(E h=$ 2-ethylhexyl $)$

1) $2 \mathrm{a}$ or $\mathbf{2 b}, \mathrm{K}_{2} \mathrm{CO}_{3}, \mathrm{DMF}^{\mathrm{a}}$

2) $\mathrm{KOH}, \mathrm{EtOH}-\mathrm{H}_{2} \mathrm{O}^{b}$

$R$<smiles></smiles>

$\mathrm{Pd}(\mathrm{OAc})_{2}{ }^{\mathrm{c}}$

$\mathrm{P}(\mathrm{o}-\mathrm{BP})(t-\mathrm{Bu})_{2}$

$\mathrm{Cs}_{2} \mathrm{CO}_{3}, \mathrm{DMAc}$

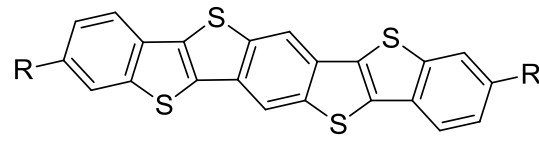

7a: $\mathrm{R}=\mathrm{H}(\mathrm{BBTBBDT})$

7b: $\mathrm{R}=n-\mathrm{C}_{8} \mathrm{H}_{17}\left(2 \mathrm{C}_{8}-\mathrm{BBTBBDT}\right)$

Scheme 3. Synthesis of BBTBBDT and $2 \mathrm{C}_{8}$-BBTBBDT. ${ }^{a}$ Conditions: [1c]::[2]: $\left[\mathrm{K}_{2} \mathrm{CO}_{3}\right]=1: 2.4: 4(3 \mathrm{mmol}$ of $\mathbf{2 a}$ and $1.2 \mathrm{mmol}$ of $\mathbf{2 b}$ were used), $80{ }^{\circ} \mathrm{C}, 18 \mathrm{~h}$ in DMF under $\mathrm{N}_{2} .{ }^{\mathrm{b}}$ Conditions: [2]:[KOH] = 1:6, $\mathrm{EtOH} / \mathrm{H}_{2} \mathrm{O}=5: 1(\mathrm{v} / \mathrm{v}), 100{ }^{\circ} \mathrm{C}, 15-21 \mathrm{~h}$ under $\mathrm{N}_{2}$. Yields of $\mathbf{6 a}$ and $\mathbf{6 b}$ were $42 \%$ and $75 \%$, respectively (by two steps). ${ }^{c}$ Conditions: $[\mathbf{6}]:\left[\mathrm{Pd}(\mathrm{OAc})_{2}\right]:[\mathrm{P}(o-$ $\left.\mathrm{BP})(t-\mathrm{Bu})_{2}\right]:\left[\mathrm{Cs}_{2} \mathrm{CO}_{3}\right]=1: 0.2: 0.4: 4(0.3 \mathrm{mmol}$ of $\mathbf{6 a}$ and $0.114 \mathrm{mmol}$ of $\mathbf{6} \mathbf{b}$ were used). Yields of $\mathbf{7 a}$ and $\mathbf{7 b}$ were $61 \%$ (18\% after sublimation in vacuo) and $26 \%$, respectively.

Butyl 3,6-bis(chloro)benzo[2,1-b:3,4- $b$ ']dithiophene-2,6carboxylate (1d) was found to be a useful scaffold for constructing an isomer of BBTBDT (iso-BBTBDT) 9a and its dioctyl derivative 9b (Scheme 4). Construction of the isoBBTBDT skeleton is to date unprecedented. The diester $\mathbf{1 d}$ can be readily prepared by the convenient double ring-closure reaction of commercially available 1,4-phenylenediacrylic acid with thionyl chloride in the presence of pyridine followed by esterification with butanol. ${ }^{11,}{ }^{15}$ Decarboxylative cyclization of the intermediary diacids $\mathbf{8 a}$ and $\mathbf{8 b}$, which were prepared from 1d with $\mathbf{2 a}$ and $\mathbf{2 b}$, gave rise to $\mathbf{9 a}$ and $\mathbf{9 b}$ in $56 \%$ and $36 \%$ yields, respectively.<smiles>CCCCOC(=O)c1sc2c(ccc3c(Cl)c(C(=O)OCC)sc32)c1Cl</smiles>
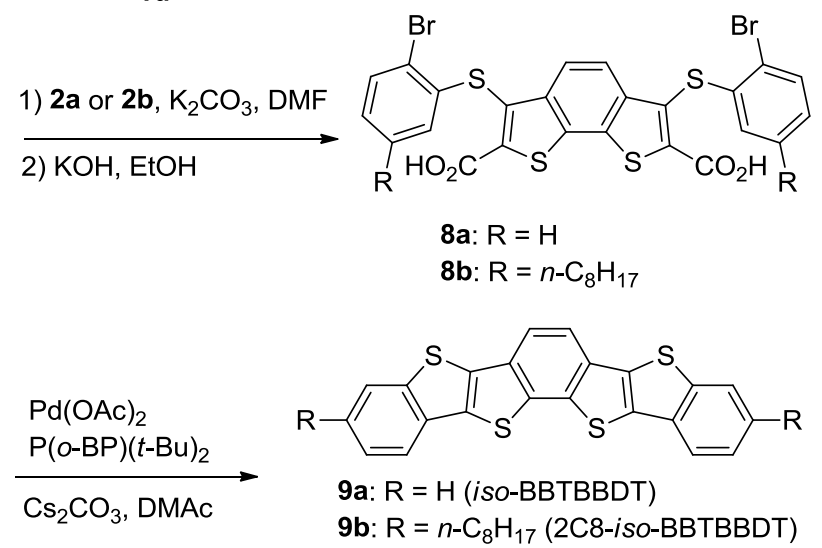

Scheme 4. Preparation of iso-BBTBBDT and $2 \mathrm{C}_{8}$-isoBBTBBDT. ${ }^{\mathrm{a} C o n d i t i o n s: ~}[\mathbf{1 d}]:[\mathbf{2}]:\left[\mathrm{K}_{2} \mathrm{CO}_{3}\right]=1: 2.4: 4(5.2 \mathrm{mmol}$ of $\mathbf{2 a}$ and $1.6 \mathrm{mmol}$ of $\mathbf{2 b}$ were used), $80{ }^{\circ} \mathrm{C}, 8 \mathrm{~h}$ in DMF under $\mathrm{N}_{2}$. ${ }^{\mathrm{b}}$ Conditions: $[2]:[\mathrm{KOH}]=1: 6, \mathrm{EtOH} / \mathrm{H}_{2} \mathrm{O}=5: 1(\mathrm{v} / \mathrm{v}), 100{ }^{\circ} \mathrm{C}, 15-21 \mathrm{~h}$. Yields of 8a and 8b were $53 \%$ and $78 \%$, respectively (by two steps). ${ }^{\circ}$ Conditions:
$[8]:\left[\mathrm{Pd}(\mathrm{OAc})_{2}\right]:\left[\mathrm{P}(o-\mathrm{BP})(t-\mathrm{Bu})_{2}\right]:\left[\mathrm{Cs}_{2} \mathrm{CO}_{3}\right]=1: 0.2: 0.4: 4(0.3 \mathrm{mmol}$ of $\mathbf{8 a}$ and $0.114 \mathrm{mmol}$ of $\mathbf{8 b}$ were used). Yields of $\mathbf{9 a}$ and $9 \mathbf{b}$ were $56 \%$ (32\% after sublimation in vacuo) and $35 \%$ (25\% after Soxhlet extraction), respectively.

In summary, we have demonstrated that 3-(chloro)- or 3(trifluoromethanesulfonyloxy)-benzo[b]thiophene-2-carboxylic acid esters in combination with 2-bromobenzenethiols are useful building-blocks for constructing BTBT and its higher homologs. Thus, by using the present strategy including palladiumcatalyzed decarboxylative arylation as the key step, not only BTBT and BBTBDT, but also a structurally new seven-ring theienoacene system, i.e. iso-BBTBDT can be constructed effectively. ${ }^{16}$ Further studies of the synthesis of related derivatives by this reaction sequence and their physical properties are underway and the details will be reported in due course.

\section{Acknowledgements}

This work was partially supported by Grants-in-Aid for Scientific Research from MEXT, JSPS, JST, Japan.

\section{Supplementary data}

Supplementary data (characterization data of compounds) associated with this article can be found in the online version at doi:

\section{References and notes}

1. (a) Recent reviews: Murphy, A. R.; Fréchet, J. M. J. Chem. Rev. 2007, 107, 1066. (b) Takimiya, K.; Shinamura, S.; Osaka, I.; Miyazaki, E.; Adv. Mater. 2011, 23, 4347 (c) Wang, C.; Dong, H.; Hu, W.; Liu, Y.; Zhu, D. Chem. Rev. 2012, 112, 2208. (d) Mei, J.; Diao, Y.; Appleton, A. L.; Fang, L.; Bao, Z. J. Am. Chem. Soc. 2013, 135, 6724. (e) Takimiya, K.; Nakano, M.; Kang, M. J.; Miyazaki, E.; Osaka, I. Eur. J. Org. Chem. 2013, 217.

2. (a) Sashida, H.; Yasuike, D. J. Heterocyclic Chem. 1998, 35, 725. (b) Li, Y.; Nie, C.; Wang, W.; Li, X.; Verpoort, F.; Duan, C. Eur. J. Org. Chem. 2011, 7331 .

3. Saito, M.; Yamamoto, T.; Osaka, I.; Miyazaki, E.; Takimiya, K.; Kuwabara, H.; Ikeda, M. Tetrahedron Lett. 2010, 51, 5277.

4. Saito, M.; Osaka, I.; Miyazaki, E.; Takimiya, K.; Kuwabara, H.; Ikeda, M. Tetrahedron Lett. 2011, 52, 285.

5. Kosta, B.; Kozmik, V.; Svoboda, J. Coll. Czech. Chem. Commun. 2002, 67, 645 .

6. Amin, A. Y.; Khassanov, A.; Reuter, K.; Meyer-Friedrichsen, T.; Halik, M. J. Am. Chem. Soc. 2012, 134, 16548.

7. Yamamoto, T.; Nishimura, T.; Mori, T.; Miyazaki, E.; Osaka, I.; Takimiya, K. Org. Lett. 2012, 14, 4914.

8. Yang, Y. S.; Yasuda, T.; Adachi, C. Bull. Chem. Soc. Jpn. 2012, $85,1186$.

9. Mori, T.; Nishimura, T.; Yamamoto, T.; Doi, I.; Miyazaki, E.; Osaka, I.; Takimiya, K. J. Am. Chem. Soc. 2013, 135, 13900.

10. (a) Satoh, T.; Miura, M. Synthesis 2010, 3395. (b) Gooßen, L. J.; Rodríguez, N.; Gooßen, K.; Angew. Chem., Int. Ed. 2008, 47, 3100 .

11. (a) Miyasaka, M.; Hirano, K.; Satoh, T.; Miura, M. Adv. Synth. Catal. 2009, 351, 2683. Corrigendum: (b) Miyasaka, M.; Hirano, K.; Satoh, T.; Miura, M. Adv. Synth. Catal. 2012, 354, 957.

12. Ota, S.; Minami, S.; Hirano, K.; Satoh, T.; Ie, Y.; Seki, S.; Aso, Y.; Miura, M. RSC Adv. 2013, 3, 12356.

13. The thiol $\mathbf{2} \mathbf{b}$ was prepared by a conventional sequence including acetylation, nitration, reduction, and Sandmeyer reaction starting from commercially available 4-octylaniline. (a) Clemens, J. J.; Davis, M. D.; Lynch, K. R.; Macdonald, T. L. Bioorg. Med. Chem. Lett., 2004, 14, 4903. (b) Cook, W. H.; Cook, K. H. J. Am. Chem. Soc., 1933, 55, 1212. (c) Agou, T.; Kobayashi, J.; Kawashima, T. Chem. Eur. J. 2007, 13, 8051.

14. Preparation of a dialkyl-BBTBDT by the sulfurization of a tetrahalo precursor with $\mathrm{S}_{8}$ was described in a patent: Watanabe, M.; Ohashi, T.; Fujita, T. Jpn. Kokai Tokkyo Koho, P2009227670A. 
15. Higa, T.; Krubsack, A. J. J. Org. Chem. 1975, 40, 3037. (b) Ried, W.; Oremek, G.; Ocakcioglu, B. Liebigs Ann. Chem. 1980, 1424. (c) Malešević, M.; Karminsky-Zamola, G.; Bajić, M.; Boykin, D. D. Heterocycles 1995, 41, 2691.

16. Dibutyl 3,6-bis[(2-bromophenyl)thio]benzo[2,1-b:3,4$\left.b^{\prime}\right]$ dithiophene-2,7-dicarboxylate (Scheme 4, the first step): In a $100 \mathrm{~mL}$ reaction flask were placed the dichloride $\mathbf{1 d}(1.0 \mathrm{~g}, 2.18$ $\mathrm{mmol})$, the thiol $\mathbf{2 a}(0.61 \mathrm{~mL}, 5.2 \mathrm{mmol}), \mathrm{K}_{2} \mathrm{CO}_{3}(1.2 \mathrm{~g}, 8.7$ $\mathrm{mmol})$, and DMF (20 mL), and the resulting mixture was stirred for $24 \mathrm{~h}$ at $80{ }^{\circ} \mathrm{C}$ under nitrogen. The reaction was quenched by adding water, extracted with chloroform, and dried over $\mathrm{Na}_{2} \mathrm{SO}_{4}$. Concentration followed by purification by column chromatography with hexane/ethyl acetate $(20: 1$, v/v) gave the diarylthiolated diester $(890 \mathrm{mg}, 1.17 \mathrm{mmol}, 53 \%)$ as a yellow solid. M.p. $134-135{ }^{\circ} \mathrm{C} ;{ }^{1} \mathrm{H}$ NMR $\left(400 \mathrm{MHz}, \mathrm{CDCl}_{3}\right) \delta 0.94(\mathrm{t}, J=$ $7.4 \mathrm{~Hz}, 6 \mathrm{H}), 1.42(\mathrm{tq}, J=7.6 \mathrm{~Hz}, 7.6 \mathrm{~Hz}, 4 \mathrm{H}), 1.70(\mathrm{tt}, J=6.8 \mathrm{~Hz}$, $6.8 \mathrm{~Hz}, 4 \mathrm{H}), 4.35(\mathrm{t}, J=6.5 \mathrm{~Hz}, 4 \mathrm{H}), 6.58-6.61(\mathrm{~m}, 2 \mathrm{H}), 6.93-$ $7.00(\mathrm{~m}, 4 \mathrm{H}), 7.52-7.55(\mathrm{~m}, 2 \mathrm{H}), 7.75(\mathrm{~s}, 2 \mathrm{H}) ;{ }^{13} \mathrm{C}$ NMR $(100$ $\left.\mathrm{MHz}, \mathrm{CDCl}_{3}\right) \delta 13.87,19.35,30.67,66.31,121.30,122.80$, $126.99,127.92,127.95,130.66,133.10,134.35,136.84,137.93$, 140.33, 161.41; HRMS (APCI) $\mathrm{m} / \mathrm{z} \quad\left([\mathrm{M}+\mathrm{H}]^{+}\right)$calcd for $\mathrm{C}_{32} \mathrm{H}_{29} \mathrm{Br}_{2} \mathrm{O}_{4} \mathrm{~S}_{4}: 764.9212$, found: 764.9227 .

3,6-Bis[(2-bromophenyl)thio]benzo[2,1-b:3,4-b']dithiophene-2,7dicarboxylic acid (8a): To a mixture of the diester $(870 \mathrm{mg}, 1.14$ $\mathrm{mmol})$ and $\mathrm{KOH}$ (384 mg, $6.84 \mathrm{mmol})$, water (2 mL), and ethanol $(10 \mathrm{~mL})$-dioxane $(4 \mathrm{~mL})$ were sequentially added, and the suspension was then heated at $100{ }^{\circ} \mathrm{C}$ for $6 \mathrm{~h}$ under nitrogen. The resulting mixture was allowed to cool to room temperature. The precipitate formed by addition of diluted aqueous $\mathrm{HCl}$ was collected and washed with water, ethanol, and hexane, and then, dried under high vacuum to provide dicarboxylic acid 8a $(755$ $\mathrm{mg}, 1.14 \mathrm{mmol}$, quant.) as a yellow solid. M.p. ca. $290{ }^{\circ} \mathrm{C}$ (decomposed); ${ }^{1} \mathrm{H}$ NMR (400 MHz, DMSO- $\left.d_{6}\right) \delta$ 6.55-6.58 (m,
2H), 7.03-7.06 (m, 4H), 7.52-7.57 (m, 2H), 7.62-7.64 (m, 2H), 14.13 (bs, $2 \mathrm{H}) ;{ }^{13} \mathrm{C}$ NMR (100 MHz, DMSO-d $) \delta 120.10$, 122.09, 127.32, 127.60, 128.33, 128.46, 132.88, 133.35, 137.14, 138.46, 139.40, 161.72; HRMS (FAB $) \mathrm{m} / \mathrm{z}\left(\mathrm{M}^{+}\right)$calcd for $\mathrm{C}_{24} \mathrm{H}_{12} \mathrm{Br}_{2} \mathrm{O}_{4} \mathrm{~S}_{4}:$ 649.7980, found: 649.7996 .

Bis[1]benzothieno[2,3-d;2',3'-d']benzo[1,2-b;5,6- $\left.b^{\prime}\right]$ dithiophene (9a): In a $20 \mathrm{~mL}$ two-necked flask were placed the diacid $8 \mathbf{8}$ (200 $\mathrm{mg}, 0.307 \mathrm{mmol}), \mathrm{Pd}(\mathrm{OAc})_{2}(14 \mathrm{mg}, 0.061 \mathrm{mmol})$ and Johnphos (37 mg, $0.123 \mathrm{mmol}$ ), $\mathrm{Cs}_{2} \mathrm{CO}_{3}(407 \mathrm{mg}, 1.23 \mathrm{mmol}$ ), and DMAc $(5 \mathrm{~mL})$. The resulting mixture was stirred for $7 \mathrm{~h}$ at $160{ }^{\circ} \mathrm{C}$ under nitrogen and cooled to room temperature. The solid in the mixture was collected by filtration and washed with water, hexane, and acetone to give 9a (69 $\mathrm{mg}, 0.171 \mathrm{mmol}, 56 \%)$ as a yellow, practically insoluble solid. This was further purified by sublimation in vacuo under heating to give the analytical sample of 9a (39.1 mg, $0.097 \mathrm{mmol}, 32 \%)$. M.p. > $300{ }^{\circ} \mathrm{C}$; HRMS (APCI) $\mathrm{m} / \mathrm{z}\left([\mathrm{M}+\mathrm{H}]^{+}\right)$calcd for $\mathrm{C}_{22} \mathrm{H}_{11} \mathrm{~S}_{4}$ : 402.9738, found: 402.9738; Anal. calcd for $\mathrm{C}_{22} \mathrm{H}_{10} \mathrm{~S}_{4}$ : C, 65.64; H, 2.50\%. Found: C, $65.41 ; \mathrm{H}, 2.63 \%$.

2,9-Dioctylbis[1]benzothieno[2,3-d;2',3'-d']benzo[1,2-b;5,6-

$b^{\prime}$ dithiophene $(\mathbf{9 b})$ : This compound was prepared as above by using $\mathbf{8 b}$ (100 mg, $0.114 \mathrm{mmol}$ ). The collected solid was washed with water, hexane, acetone, and finally with chloroform to give 9b $(24.8 \mathrm{mg}, 0.040 \mathrm{mmol}, 35 \%)$ as a sparingly soluble yellow solid. A further purification was made by Soxhlet extraction with chlorobenzene to afford a somewhat clear colored sample of $\mathbf{9 b}$ (17.5 mg, $0.028 \mathrm{mmol} 25 \%$ ). M.p. ca. $210{ }^{\circ} \mathrm{C}$ (decomposed); ${ }^{1} \mathrm{H}$ NMR (400 MHz, toluene- $d_{8}$ at $\left.100{ }^{\circ} \mathrm{C}\right) \delta 0.90(\mathrm{t}, J=6.4 \mathrm{~Hz}, 6 \mathrm{H})$, $1.24-1.42(\mathrm{~m}, 20 \mathrm{H}), 1.63(\mathrm{tt}, J=6.4 \mathrm{~Hz}, 6.4 \mathrm{~Hz}, 4 \mathrm{H}), 2.61(\mathrm{t}, J=$ 7.4, 4H), 7.49 (s, 2H), 7.583 (s, 2H), 7.585 (d, $J=6.0 \mathrm{~Hz}, 2 \mathrm{H})$ (one signal was overlapped by the solvent signal); HRMS (APCI) $\mathrm{m} / \mathrm{z}\left([\mathrm{M}+\mathrm{H}]^{+}\right)$calcd for $\mathrm{C}_{38} \mathrm{H}_{43} \mathrm{~S}_{4}: 627.2244$, found: 627.2242. 
Synthesis of [1]benzothieno[3,2-b][1]benzothiophene (BTBT) and its higher homologs through palladium-catalyzed intramolecular decarboxylative arylation

Sojiro Minami ${ }^{\mathrm{a}}$, Koji Hirano ${ }^{\mathrm{a}}$, Satoh Tetsuya ${ }^{\mathrm{a}, \mathrm{b}}$, Masahiro Miura ${ }^{\mathrm{a}, *}$

${ }^{a}$ Department of Applied Chemistry, Faculty of Engineering, Osaka University, Suita, Osaka 565-0871, Japan

bJST, ACT-C, 4-1-8 Honcho, Kawaguchi, Saitama 332-0012, Japan

\section{Supplementary data}

Characterization data for compounds............... S1

NMR spectra of compounds......................S5

\section{Characterization data for compounds.}

Methyl 3-[(2-bromophenyl)thio]benzo[b]thiophene-2-carboxylate: light yellow solid; $\operatorname{NMR}\left(400 \mathrm{MHz}, \mathrm{CDCl}_{3}\right) \delta 3.92(\mathrm{~s}, 3 \mathrm{H}), 6.61(\mathrm{dd}, J=7.2 \mathrm{~Hz}, 2.1 \mathrm{~Hz}, 1 \mathrm{H}), 6.94-7.01(\mathrm{~m}, 2 \mathrm{H})$, $7.38(\mathrm{dd}, J=7.6 \mathrm{~Hz}, 7.6 \mathrm{~Hz}, 1 \mathrm{H}), 7.50(\mathrm{dd}, J=7.6 \mathrm{~Hz}, 7.6 \mathrm{~Hz}, 1 \mathrm{H}), 7.55$ (dd, $J=7.2 \mathrm{~Hz}, 2.1$ $\mathrm{Hz}, 1 \mathrm{H}), 7.84(\mathrm{~d}, J=8.1 \mathrm{~Hz}, 1 \mathrm{H}), 7.89(\mathrm{~d}, J=8.1 \mathrm{~Hz}, 1 \mathrm{H}) ;{ }^{13} \mathrm{C} \mathrm{NMR}\left(100 \mathrm{MHz}, \mathrm{CDCl}_{3}\right) \delta$ 52.86, 121.47, 122.93, 125.42, 125.66, 126.87, 127.87, 127.89, 128.26, 130.01, 133.03, 136.21, 138.22, 140.07, 140.13, 162.13; HRMS (APCI) m/z $\left(\mathrm{M}^{+}\right)$calcd for $\mathrm{C}_{16} \mathrm{H}_{11} \mathrm{BrO}_{2} \mathrm{~S}_{2}$ : 379.9358, found: 379.9366 .

3-[(2-Bromophenyl)thio]benzo[b]thiophene-2-carboxylic acid (3a): light yellow solid; m.p. $207{ }^{\circ} \mathrm{C} ;{ }^{1} \mathrm{H}$ NMR $\left(400 \mathrm{MHz}, \mathrm{DMSO}-d_{6}\right) \delta 6.54(\mathrm{dd}, J=7.6 \mathrm{~Hz}, 1.2 \mathrm{~Hz}, 1 \mathrm{H}), 7.07(\mathrm{ddd}, J=$ $7.6 \mathrm{~Hz}, 7.6 \mathrm{~Hz}, 1.2 \mathrm{~Hz}, 1 \mathrm{H}), 7.13$ (ddd, $J=7.6 \mathrm{~Hz}, 7.6 \mathrm{~Hz}, 1.2 \mathrm{~Hz}, 1 \mathrm{H}), 7.45$ (dd, $J=7.6 \mathrm{~Hz}$, $7.6 \mathrm{~Hz}, 1 \mathrm{H}), 7.57$ (dd, $J=7.6 \mathrm{~Hz}, 7.6 \mathrm{~Hz}, 1 \mathrm{H}), 7.66(\mathrm{~d}, J=8.1 \mathrm{~Hz}, 2 \mathrm{H}), 8.15(\mathrm{~d}, J=8.1 \mathrm{~Hz}$, $1 \mathrm{H}), 13.91$ (bs, $1 \mathrm{H}) ;{ }^{13} \mathrm{C}$ NMR $\left(100 \mathrm{MHz}, \mathrm{DMSO}-d_{6}\right) \delta 120.16,123.60,124.12,125.87$, $126.85,127.22$, 127.56, 127.83, 128.43, 132.87, 137.35, 138.68, 139.22, 139.27, 162.29; HRMS (FAB $\left.{ }^{-}\right) \mathrm{m} / \mathrm{z}\left([\mathrm{M}-\mathrm{H}]^{+}\right)$calcd for $\mathrm{C}_{15} \mathrm{H}_{8} \mathrm{BrO}_{2} \mathrm{~S}_{2}: 362.9143$, found: 362.9139 .

[1]Benzothieno[3,2-b][1] benzothiophene (5a): white solid; ${ }^{1} \mathrm{H} \mathrm{NMR}\left(400 \mathrm{MHz}, \mathrm{CDCl}_{3}\right) \delta$ 7.37-7.50 (m, 4H), 7.86-7.95 (m, 4H); $\left.{ }^{13} \mathrm{C} \mathrm{NMR} \mathrm{(100} \mathrm{MHz,} \mathrm{CDCl}_{3}\right) \delta 121.75,124.19$, $125.03,125.16,133.26,133.59,142.42$.

Methyl 3-[(2-bromo-5-octylphenyl)thio]benzo[b]thiophene-2-carboxylate: light yellow solid; m.p. 55-56 ${ }^{\circ} \mathrm{C} ;{ }^{1} \mathrm{H}$ NMR $\left(400 \mathrm{MHz}, \mathrm{CDCl}_{3}\right) \delta 0.86(\mathrm{t}, J=7.1 \mathrm{~Hz}, 3 \mathrm{H}), 0.94-1.32(\mathrm{~m}, 12$ H), $2.25(\mathrm{t}, J=7.5 \mathrm{~Hz}, 2 \mathrm{H}), 3.93(\mathrm{~s}, 3 \mathrm{H}), 6.42(\mathrm{~d}, J=2.1 \mathrm{z}, 1 \mathrm{H}), 6.78(\mathrm{dd}, J=8.1 \mathrm{~Hz}, 2.1 \mathrm{~Hz}$, 
1H), 7.34 (ddd, $J=8.2 \mathrm{~Hz}, 7.1 \mathrm{~Hz}, 1.2 \mathrm{~Hz}, 1 \mathrm{H}), 7.43$ (d, $J=8.1 \mathrm{~Hz}, 1 \mathrm{H}), 7.48$ (ddd, $J=8.2$ $\mathrm{Hz}, 7.1 \mathrm{~Hz}, 1.2 \mathrm{~Hz}, 1 \mathrm{H}), 7.76(\mathrm{dd}, J=8.1 \mathrm{~Hz}, 1.2 \mathrm{~Hz}, 1 \mathrm{H}), 7.88(\mathrm{dd}, J=8.1 \mathrm{~Hz}, 1.2 \mathrm{~Hz}, 1 \mathrm{H})$; ${ }^{13} \mathrm{C}$ NMR $\left(100 \mathrm{MHz}, \mathrm{CDCl}_{3}\right) \delta 14.25,22.77,28.78,29.22,29.34,30.93,31.98,35.17,52.84$, 118.62 , 122.88, 125.47, 125.51, 127.52, 127.76, 128.67, 130.66, 132.73, 135.33, 137.24, 139.84, 140.03, 142.84, 162.24; HRMS (APCI) m/z $\left(\mathrm{M}^{+}\right.$) calcd for $\mathrm{C}_{24} \mathrm{H}_{27} \mathrm{BrO}_{2} \mathrm{~S}_{2}$ : 492.0610, found: 492.0604 .

3-[(2-Bromo-5-octylphenyl)thio]benzo[b]thiophene-2-carboxylic acid (3b): yellow solid; m.p. $142-143{ }^{\circ} \mathrm{C} ;{ }^{1} \mathrm{H}$ NMR (400 MHz, $\left.\mathrm{CDCl}_{3}\right) \delta 0.85(\mathrm{t}, J=7.1 \mathrm{~Hz}, 3 \mathrm{H}), 0.96-1.34(\mathrm{~m}, 12 \mathrm{H})$, $2.27(\mathrm{t}, J=7.5 \mathrm{~Hz}, 2 \mathrm{H}), 6.44(\mathrm{~d}, J=2.1 \mathrm{~Hz}, 1 \mathrm{H}), 6.82(\mathrm{dd}, J=8.1 \mathrm{~Hz}, 2.1 \mathrm{~Hz}, 1 \mathrm{H}), 7.37$ (ddd, $J=8.2 \mathrm{~Hz}, 7.1 \mathrm{~Hz}, 1.0 \mathrm{~Hz}, 1 \mathrm{H}), 7.45(\mathrm{~d}, J=8.1 \mathrm{~Hz}, 1 \mathrm{H}), 7.51$ (ddd, $J=8.2 \mathrm{~Hz}, 7.1 \mathrm{~Hz}, 1.2$ $\mathrm{Hz}, 1 \mathrm{H}), 7.76(\mathrm{dd}, J=8.1 \mathrm{~Hz}, 1.0 \mathrm{~Hz}, 1 \mathrm{H}), 7.91(\mathrm{dd}, J=8.1 \mathrm{~Hz}, 1.0 \mathrm{~Hz}, 1 \mathrm{H}) ;{ }^{13} \mathrm{C} \mathrm{NMR}(100$ $\left.\mathrm{MHz} \mathrm{CDCl}_{3}\right) \delta 14.24,22.77,28.81,29.22,29.38,30.90,31.97,35.19,118.96,123.05$, $125.63,125.70,128.08,128.23,128.94,131.28,132.94,135.30,136.14,139.77,140.69$, 143.17, 165.79; HRMS (APCI) $\mathrm{m} / \mathrm{z}\left([\mathrm{M}-\mathrm{H}]^{+}\right)$calcd for $\mathrm{C}_{23} \mathrm{H}_{24} \mathrm{BrO}_{2} \mathrm{~S}_{2}$ : 475.0395, found: 475.0407

2-Octyl-[1]benzothieno[3,2-b][1]benzothiophene (5b): colorless solid; m.p. $107-108{ }^{\circ} \mathrm{C} ;{ }^{1} \mathrm{H}$ NMR (400 MHz, $\left.\mathrm{CDCl}_{3}\right) \delta 0.90(\mathrm{t}, J=7.1 \mathrm{~Hz}, 3 \mathrm{H}), 1.22-1.45(\mathrm{~m}, 10 \mathrm{H}), 1.71(\mathrm{tt}, J=7.6 \mathrm{~Hz}$, $7.6 \mathrm{~Hz}, 2 \mathrm{H}), 2.76(\mathrm{t}, J=7.6 \mathrm{~Hz}, 2 \mathrm{H}), 7.28(\mathrm{~d}, J=8.1 \mathrm{~Hz}, 1 \mathrm{H}), 7.39(\mathrm{dd}, J=7.9 \mathrm{~Hz}, 7.9 \mathrm{~Hz}$, 1H), 7.45 (dd, $J=7.9 \mathrm{~Hz}, 7.9 \mathrm{~Hz}, 1 \mathrm{H}), 7.72$ (s, 1H), 7.79 (d, $J=8.1 \mathrm{~Hz}, 1 \mathrm{H}), 7.87$ (d, $J=7.9$ $\mathrm{Hz}, 1 \mathrm{H}), 7.91(\mathrm{~d}, J=7.9 \mathrm{~Hz}, 1 \mathrm{H}) ;{ }^{13} \mathrm{C} \mathrm{NMR}\left(100 \mathrm{MHz}, \mathrm{CDCl}_{3}\right) \delta 14.26,22.82,29.42,29.48$, 29.64, 31.84, 32.04, 36.28, 121.39, 121.54, 123.49, 124.13, 124.84, 124.94, 126.04, 131.16, $132.73,133.42,133.54,140.54,142.23,142.73$; HRMS (APCI) $\mathrm{m} / \mathrm{z}\left([\mathrm{M}+\mathrm{H}]^{+}\right)$calcd for $\mathrm{C}_{22} \mathrm{H}_{25} \mathrm{~S}_{2}: 353.1392$, found: 353.1393 .

\section{Bis(2-ethylhexyl)}

3,7-bis[(2-bromophenyl)thio]benzo[1,2-b:4,5- $\left.b^{\prime}\right]$ dithiophene2,6-dicarboxylate: yellow solid; m.p. $139-140{ }^{\circ} \mathrm{C} ;{ }^{1} \mathrm{H}$ NMR $\left(400 \mathrm{MHz}, \mathrm{CDCl}_{3}\right) \delta 0.86(\mathrm{t}, J=$ $7.4 \mathrm{~Hz}, 12 \mathrm{H}), 1.20-1.42(\mathrm{~m}, 16 \mathrm{H}), 1.62(\mathrm{tt}, J=6.1 \mathrm{~Hz}, 6.1 \mathrm{~Hz}, 2 \mathrm{H}), 4.23$ (dd, $J=11 \mathrm{~Hz}, 5.2$ $\mathrm{Hz}, 2 \mathrm{H}), 4.27$ (dd, $J=11 \mathrm{~Hz}, 5.2 \mathrm{~Hz}, 2 \mathrm{H}), 6.53-6.56(\mathrm{~m}, 2 \mathrm{H}), 6.96-7.01(\mathrm{~m}, 4 \mathrm{H}), 7.56-7.58$ (m, 2H), 8.44 (s, 2H); ${ }^{13} \mathrm{C} \mathrm{NMR}\left(100 \mathrm{MHz}, \mathrm{CDCl}_{3}\right) \delta 11.22,14.21,23.06,23.93,29.07,30.49$, $38.90,68.75,119.47,120.92,126.83,127.28,127.34,128.00,133.14,137.63,137.87,140.48$, 140.88, 161.75; HRMS (APCI) $\mathrm{m} / \mathrm{z}\left(\mathrm{M}^{+}\right)$calcd for $\mathrm{C}_{40} \mathrm{H}_{44} \mathrm{Br}_{2} \mathrm{O}_{4} \mathrm{~S}_{4}$ : 876.0466, found: 876.0465 .

3,7-Bis[(2-bromophenyl)thio]benzo[1,2-b:4,5- $\left.b^{\prime}\right]$ dithiophene-2,6-dicarboxylic acid (6a): deep yellow solid; m.p. > $300{ }^{\circ} \mathrm{C} ;{ }^{1} \mathrm{H}$ NMR (400 MHz, DMSO- $\left.d_{6}\right) \delta 6.53$ (dd, $J=7.8 \mathrm{~Hz}, 1.7$ $\mathrm{Hz}, 2 \mathrm{H}$ ), 7.07 (ddd, $J=7.5 \mathrm{~Hz}, 7.5 \mathrm{~Hz}, 1.7 \mathrm{~Hz}, 2 \mathrm{H}$ ), 7.13 (ddd, $J=7.8 \mathrm{~Hz}, 7.8 \mathrm{~Hz}, 1.5 \mathrm{~Hz}$, 2H), 7.67 (dd, $J=7.5 \mathrm{~Hz}, 1.5 \mathrm{~Hz}, 2 \mathrm{H}), 8.63(\mathrm{~s}, 2 \mathrm{H}), 14.05(\mathrm{bs}, 2 \mathrm{H}) ;{ }^{13} \mathrm{C}$ NMR (100 MHz, 
DMSO- $\left.d_{6}\right) \delta 119.19,119.78,125.13,126.80,127.01,128.51,132.87,136.91,137.43,139.86$, 142.34, 161.99; HRMS (FAB $\left.{ }^{-}\right) \mathrm{m} / \mathrm{z}\left(\mathrm{M}^{+}\right)$calcd for $\mathrm{C}_{24} \mathrm{H}_{12} \mathrm{Br}_{2} \mathrm{O}_{4} \mathrm{~S}_{4}$ : 649.7980, found: 649.7982 .

Bis[1]benzothieno[2,3- $\left.d ; 2^{\prime}, 3^{\prime}-d^{\prime}\right]$ benzo[1,2-b;4,5-b']dithiophene $\quad(7 a): \quad$ yellow solid (practically insoluble); m.p. > $300{ }^{\circ} \mathrm{C}$; HRMS (APCI) m/z $\left([\mathrm{M}+\mathrm{H}]^{+}\right)$calcd for $\mathrm{C}_{22} \mathrm{H}_{11} \mathrm{~S}_{4}$ : 402.9738, found: 402.9736; Anal. calcd for $\mathrm{C}_{22} \mathrm{H}_{10} \mathrm{~S}_{4}$ : C, 65.64; H, 2.50\%. Found: C, 65.17; H, $2.60 \%$.

Bis(2-ethylhexyl) 3,7-bis[(2-bromo-5-octylphenyl)thio]benzo[1,2- $b$ :4,5- $\left.b^{\prime}\right]$ dithiophene2,6-dicarboxylate: yellow solid; m.p. $63-64{ }^{\circ} \mathrm{C} ;{ }^{1} \mathrm{H}$ NMR $\left(400 \mathrm{MHz}, \mathrm{CDCl}_{3}\right) \delta 0.84(\mathrm{t}, J=$ $7.1 \mathrm{~Hz}, 6 \mathrm{H}), 0.866(\mathrm{t}, J=6.9 \mathrm{~Hz}, 6 \mathrm{H}), 0.871(\mathrm{t}, J=7.4 \mathrm{~Hz}, 6 \mathrm{H}), 0.94-1.44(\mathrm{~m}, 40 \mathrm{H}), 1.63$ (tt, $J=6.0 \mathrm{~Hz}, 6.0 \mathrm{~Hz}, 2 \mathrm{H}), 2.23(\mathrm{t}, J=7.5 \mathrm{~Hz}, 4 \mathrm{H}), 4.23(\mathrm{dd}, J=11 \mathrm{~Hz}, 5.4 \mathrm{~Hz}, 2 \mathrm{H}), 4.28(\mathrm{dd}, J$ $=11 \mathrm{~Hz}, 5.4 \mathrm{~Hz}, 2 \mathrm{H}), .6 .37(\mathrm{~d}, J=2.0 \mathrm{~Hz}, 2 \mathrm{H}), 6.79(\mathrm{dd}, J=8.1 \mathrm{~Hz}, 2.0 \mathrm{~Hz}, 2 \mathrm{H}), 7.45(\mathrm{~d}, J=$ $8.1 \mathrm{~Hz}, 2 \mathrm{H}), 8.36(\mathrm{~s}, 2 \mathrm{H}) ;{ }^{13} \mathrm{C} \mathrm{NMR}\left(100 \mathrm{MHz}, \mathrm{CDCl}_{3}\right) \delta 11.21,14.21,12.25,22.76,23.07$, 23.94, 28.86, 29.09, 29.20, 29.38, 30.51, 30.99, 31.98, 35.23, 38.93, 68.70, 118.15, 119.55, $127.45,127.73$, 127.99, 132.90, 136.94, 137.47, 140.06, 140.21, 143.01, 161.84; HRMS (APCI) $\mathrm{m} / \mathrm{z}\left(\mathrm{M}^{+}\right)$calcd for $\mathrm{C}_{56} \mathrm{H}_{76} \mathrm{Br}_{2} \mathrm{O}_{4} \mathrm{~S}_{4}:$ 1100.2973, found: 1100.2972 .

\section{3,7-Bis[(2-bromo-5-octylphenyl)thio]benzo[1,2-b:4,5-b']dithiophene-2,6-dicarboxylic}

acid (6b): yellow solid; ca. $260{ }^{\circ} \mathrm{C}$ (decomposed); ${ }^{1} \mathrm{H}$ NMR (400 MHz, DMSO- $\left.d_{6}\right) \delta$ 0.70-1.18 (m, 30H), 2.20 (t, $J=7.0 \mathrm{~Hz}, 4 \mathrm{H}), 6.27$ (d, $J=1.9 \mathrm{~Hz}, 2 \mathrm{H}), 6.89$ (dd, $J=8.1 \mathrm{~Hz}$, $1.9 \mathrm{~Hz}, 2 \mathrm{H}), 7.56(\mathrm{~d}, J=8.1 \mathrm{~Hz}, 2 \mathrm{H}), 8.40(\mathrm{~s}, 2 \mathrm{H}) ;{ }^{13} \mathrm{C} \mathrm{NMR}\left(100 \mathrm{MHz}, \mathrm{CDCl}_{3}\right) \delta 13.56$, $21.67,27.49,28.04,28.34,29.76,30.95,33.80,117.14,118.81,125.55,127.17,127.40$, $132.46,136.23,136.42,138.96,141.13,142.28,161.74$.

2,9-Dioctylbis[1]benzothieno[2,3- $\left.d ; 2^{\prime}, 3^{\prime}-d^{\prime}\right]$ benzo[1,2-b;4,5- $\left.b^{\prime}\right]$ dithiophene (7b) yellow solid (sparingly soluble); ca. $260{ }^{\circ} \mathrm{C}$ (decomposed); ${ }^{1} \mathrm{H}$ NMR $\left(400 \mathrm{MHz}\right.$, toluene- $d_{8}, 100{ }^{\circ} \mathrm{C}$ ) $\delta 0.89(\mathrm{t}, J=6.8 \mathrm{~Hz}, 6 \mathrm{H}), 1.24-1.37(\mathrm{~m}, 20 \mathrm{H}), 1.62(\mathrm{tt}, J=7.0 \mathrm{~Hz}, 7.0 \mathrm{~Hz}, 4 \mathrm{H}), 2.60(\mathrm{t}, J=$ $7.4 \mathrm{~Hz}, 4 \mathrm{H}), 7.08$ (d, $J=8.0 \mathrm{~Hz}, 2 \mathrm{H}), 7.50$ (s, 2H), 7.59 (d, $J=8.0 \mathrm{~Hz}, 2 \mathrm{H}), 7.96$ (s, 2H); HRMS (APCI) m/z $\left([\mathrm{M}+\mathrm{H}]^{+}\right)$calcd for $\mathrm{C}_{38} \mathrm{H}_{43} \mathrm{~S}_{2}: 627.2244$, found: 627.2242.

Dibutyl 3,6-bis[(2-bromophenyl)thio]benzo[2,1-b:3,4- $\left.b^{\prime}\right]$ dithiophene-2,7-dicarboxylate: yellow solid; m.p. $134-135{ }^{\circ} \mathrm{C} ;{ }^{1} \mathrm{H}$ NMR $\left(400 \mathrm{MHz}, \mathrm{CDCl}_{3}\right) \delta 0.94(\mathrm{t}, J=7.4 \mathrm{~Hz}, 6 \mathrm{H}), 1.42$ (tq, $J=7.6 \mathrm{~Hz}, 7.6 \mathrm{~Hz}, 4 \mathrm{H}), 1.70(\mathrm{tt}, J=6.8 \mathrm{~Hz}, 6.8 \mathrm{~Hz}, 4 \mathrm{H}), 4.35$ (t, $J=6.5 \mathrm{~Hz}, 4 \mathrm{H})$, 6.58-6.61 (m, 2H), 6.93-7.00 (m, 4H), 7.52-7.55 (m, 2H), $7.75(\mathrm{~s}, 2 \mathrm{H}) ;{ }^{13} \mathrm{C}$ NMR (100 MHz, $\left.\mathrm{CDCl}_{3}\right) \delta 13.87,19.35,30.67,66.31,121.30,122.80,126.99,127.92,127.95,130.66,133.10$, 134.35, 136.84, 137.93, 140.33, 161.41; HRMS (APCI) $\mathrm{m} / \mathrm{z} \quad\left([\mathrm{M}+\mathrm{H}]^{+}\right)$calcd for $\mathrm{C}_{32} \mathrm{H}_{29} \mathrm{Br}_{2} \mathrm{O}_{4} \mathrm{~S}_{4}:$ 764.9212, found: 764.9227. 
3,6-Bis[(2-bromophenyl)thio]benzo[2,1-b:3,4- $\left.b^{\prime}\right]$ dithiophene-2,7-dicarboxylic acid (8a): yellow solid; m.p. ca. $290{ }^{\circ} \mathrm{C}$ (decomposed); ${ }^{1} \mathrm{H}$ NMR (400 MHz, DMSO- $\left.d_{6}\right) \delta$ 6.55-6.58 (m, 2H), 7.03-7.06 (m, 4H), 7.52-7.57 (m, 2H), 7.62-7.64 (m, 2H), 14.13 (bs, 2H); ${ }^{13} \mathrm{C} \mathrm{NMR} \mathrm{(100}$ MHz, DMSO- $\left.d_{6}\right) \delta 120.10,122.09,127.32,127.60,128.33,128.46,132.88,133.35,137.14$, 138.46, 139.40, 161.72; HRMS (FAB $) \mathrm{m} / \mathrm{z}\left(\mathrm{M}^{+}\right)$calcd for $\mathrm{C}_{24} \mathrm{H}_{12} \mathrm{Br}_{2} \mathrm{O}_{4} \mathrm{~S}_{4}$ : 649.7980, found: 649.7996.

Bis[1]benzothieno[2,3- $\left.d ; 2^{\prime}, 3^{\prime}-d^{\prime}\right]$ benzo[1,2-b;5,6-b']dithiophene $\quad(9 a)$ yellow solid (practically insolubule); m.p. > $300{ }^{\circ} \mathrm{C}$; HRMS (APCI) $\mathrm{m} / \mathrm{z}\left([\mathrm{M}+\mathrm{H}]^{+}\right.$) calcd for $\mathrm{C}_{22} \mathrm{H}_{11} \mathrm{~S}_{4}$ : 402.9738, found: 402.9738; Anal. calcd for $\mathrm{C}_{22} \mathrm{H}_{10} \mathrm{~S}_{4}$ : C, 65.64; H, 2.50\%. Found: C, 65.41; H, $2.63 \%$.

Dibutyl

3,6-bis[(2-bromo-5-octylphenyl)thio]benzo[2,1-b:3,4- $b$ ']dithiophene2,7-dicarboxylate: yellow solid; m.p. $102-103{ }^{\circ} \mathrm{C} ;{ }^{1} \mathrm{H}$ NMR $\left(400 \mathrm{MHz}, \mathrm{CDCl}_{3}\right) \delta 0.84$ (t, $J=$ $7.0 \mathrm{~Hz}, 6 \mathrm{H}), 0.94(\mathrm{t}, J=7.4 \mathrm{~Hz}, 6 \mathrm{H}), 0.96-1.32(\mathrm{~m}, 24 \mathrm{H}), 1.42(\mathrm{tq}, J=7.6 \mathrm{~Hz}, 7.6 \mathrm{~Hz}, 4 \mathrm{H})$, $1.70(\mathrm{tt}, J=6.8 \mathrm{~Hz}, 6.8 \mathrm{~Hz}, 4 \mathrm{H}), 2.24(\mathrm{t}, J=7.6 \mathrm{~Hz}, 7.6 \mathrm{~Hz}, 4 \mathrm{H}), 4.35(\mathrm{t}, J=6.6 \mathrm{~Hz}, 4 \mathrm{H}), 6.44$ $(\mathrm{d}, J=2.0 \mathrm{~Hz}, 2 \mathrm{H}), 6.78(\mathrm{dd}, J=8.1 \mathrm{~Hz}, 2.0 \mathrm{~Hz}, 2 \mathrm{H}), 7.42(\mathrm{~d}, J=8.1 \mathrm{~Hz}, 2 \mathrm{H}), 7.69(\mathrm{~s}, 2 \mathrm{H})$; ${ }^{13} \mathrm{C}$ NMR $\left(100 \mathrm{MHz}, \mathrm{CDCl}_{3}\right) \delta 13.87,14.26,19.36,22.77,28.85,29.23,29.37,30.71,30.96$, 31.98, 35.19, 66.24, 118.56, 122.76, 127.64, 128.40, 131.36, 132.86, 134.28, 136.03, 137.05, 140.20, 142.99, 161.53; HRMS (APCI) $\mathrm{m} / \mathrm{z}\left([\mathrm{M}+\mathrm{H}]^{+}\right)$calcd for $\mathrm{C}_{48} \mathrm{H}_{61} \mathrm{Br}_{2} \mathrm{O}_{4} \mathrm{~S}_{4}: 987.1798$, found: 987.1792 .

\section{3,6-Bis((2-bromo-5-octylphenyl)thio)benzo[2,1-b:3,4-b']dithiophene-2,7-dicarboxylic}

acid (8b): yellow solid; m.p. 232-233 ${ }^{\circ} \mathrm{C}$; ${ }^{1} \mathrm{H}$ NMR (400 MHz, DMSO- $\left.d_{6}\right) \delta 0.78$ (t, $J=7.1$ $\mathrm{Hz}, 6 \mathrm{H}), 0.68-1.20(\mathrm{~m}, 24 \mathrm{H}), 2.19(\mathrm{t}, J=7.1 \mathrm{~Hz}, 7.1 \mathrm{~Hz}, 4 \mathrm{H}), 6.36(\mathrm{~d}, J=2.0 \mathrm{~Hz}, 2 \mathrm{H}), 6.87$ $(\mathrm{dd}, J=8.1 \mathrm{~Hz}, 2.0 \mathrm{~Hz}, 2 \mathrm{H}), 7.51(\mathrm{~d}, J=8.1 \mathrm{~Hz}, 2 \mathrm{H}), 7.69(\mathrm{~m}, 2 \mathrm{H}) ;{ }^{13} \mathrm{C} \mathrm{NMR}(100 \mathrm{MHz}$, DMSO- $\left.d_{6}\right) \delta 13.97,22.06,27.62,28.38,28.53,29.89,31.30,33.80,117.47,121.95,127.70$, 127.83, 129.00, 132.68, 133.22, 136.39, 137.72, 139.17, 142.55, 161.76; HRMS $\left(\mathrm{FAB}^{+}\right) \mathrm{m} / \mathrm{z}$ $\left(\mathrm{M}^{+}\right)$calcd for $\mathrm{C}_{40} \mathrm{H}_{44} \mathrm{Br}_{2} \mathrm{O}_{4} \mathrm{~S}_{4}:$ 874.0469, found: 874.0467.

2,9-Dioctylbis[1]benzothieno[2,3- $\left.d ; 2^{\prime}, 3^{\prime}-d^{\prime}\right]$ benzo[1,2-b;5,6-b']dithiophene (9b): yellow solid (sparingly soluble); m.p. ca. $210{ }^{\circ} \mathrm{C}$ (decomposed); ${ }^{1} \mathrm{H}$ NMR (400 MHz, toluene- $d_{8}$ at $\left.100{ }^{\circ} \mathrm{C}\right) \delta 0.90(\mathrm{t}, J=6.4 \mathrm{~Hz}, 6 \mathrm{H}), 1.24-1.42(\mathrm{~m}, 20 \mathrm{H}), 1.63(\mathrm{tt}, J=6.4 \mathrm{~Hz}, 6.4 \mathrm{~Hz}, 4 \mathrm{H}), 2.61$ (t, $J=7.4,4 \mathrm{H}), 7.49(\mathrm{~s}, 2 \mathrm{H}), 7.583(\mathrm{~s}, 2 \mathrm{H}), 7.585(\mathrm{~d}, J=6.0 \mathrm{~Hz}, 2 \mathrm{H})$ (one signal was overlapped by the solvent signal); HRMS (APCI) $\mathrm{m} / \mathrm{z}\left([\mathrm{M}+\mathrm{H}]^{+}\right.$) calcd for $\mathrm{C}_{38} \mathrm{H}_{43} \mathrm{~S}_{4}: 627.2244$, found: 627.2242 . 
$\left[{ }^{1} \mathrm{H}\right.$ and ${ }^{13} \mathrm{C}$ NMR Spectra of Methyl Ester of 3a]
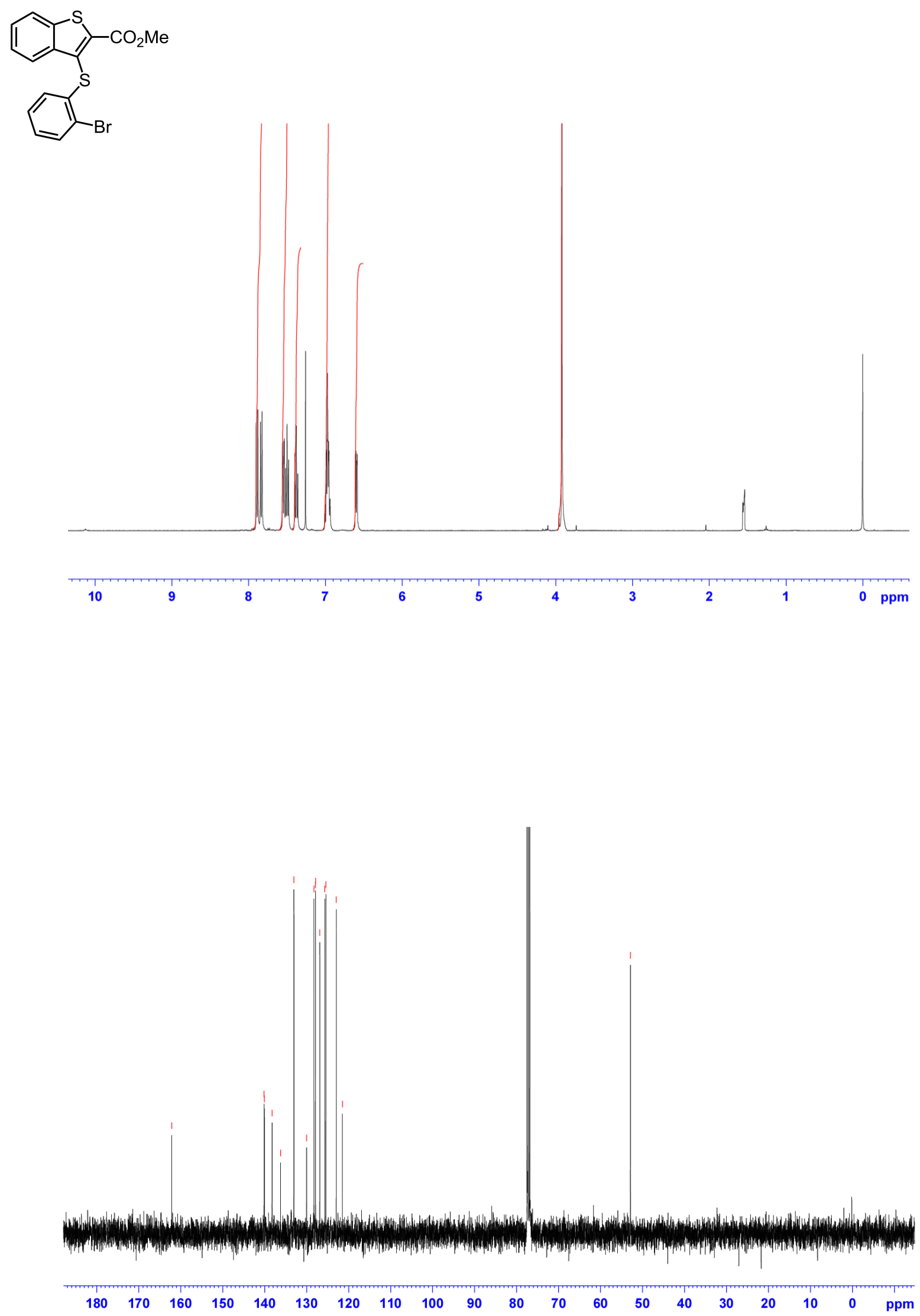
$\left[{ }^{1} \mathrm{H}\right.$ and ${ }^{13} \mathrm{C}$ NMR Spectra of 3a]
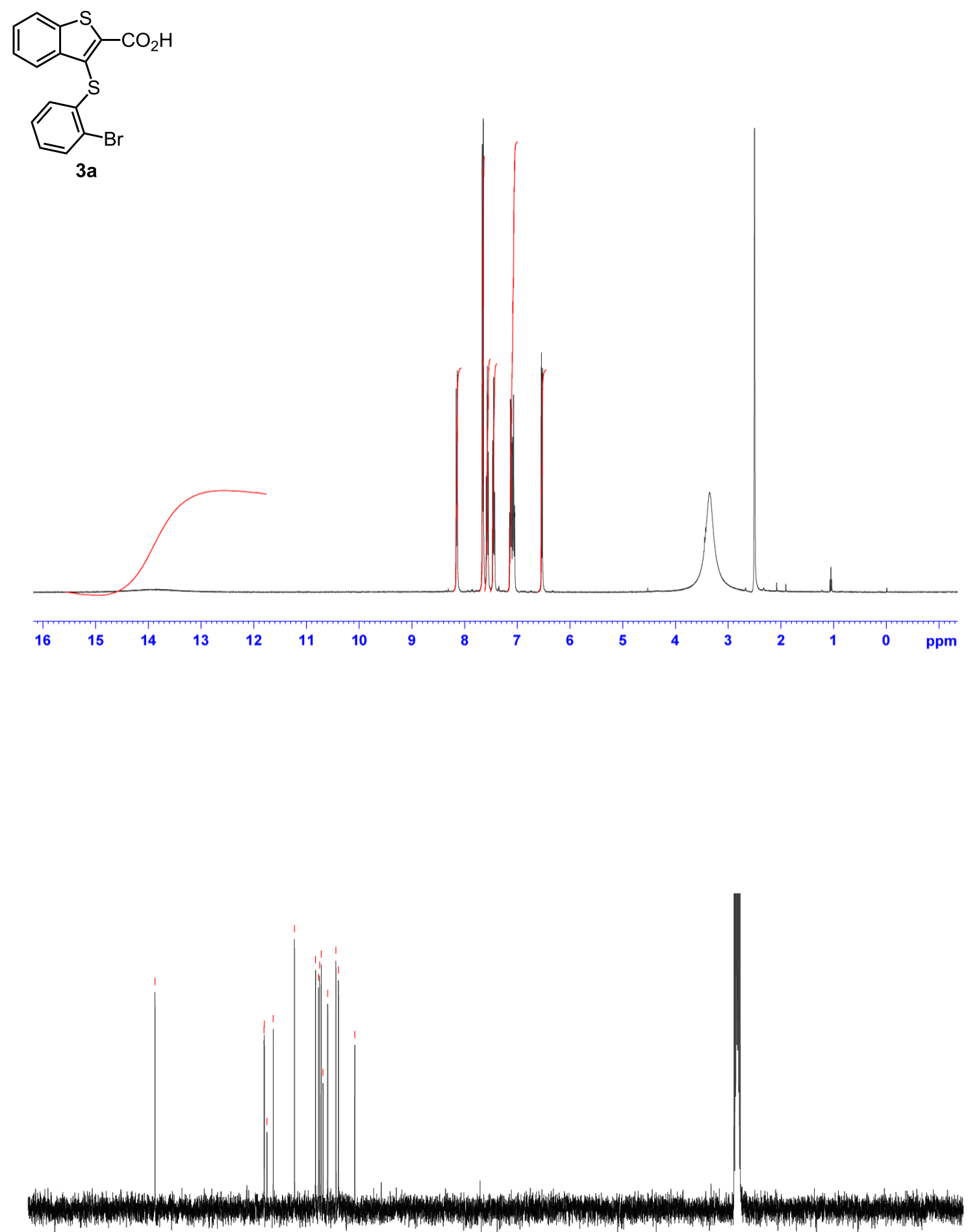

$\begin{array}{lllllllllllllllllll}180 & 170 & 160 & 150 & 140 & 130 & 120 & 110 & 100 & 90 & 80 & 70 & 60 & 50 & 40 & 30 & 20 & 10 & \mathrm{ppm}\end{array}$ 
$\left[{ }^{1} \mathrm{H}\right.$ and ${ }^{13} \mathrm{C}$ NMR Spectra of 5a]

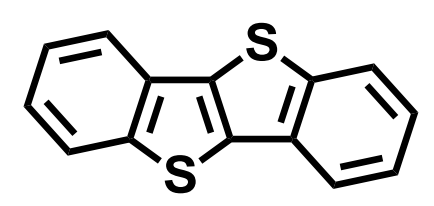

$5 a$
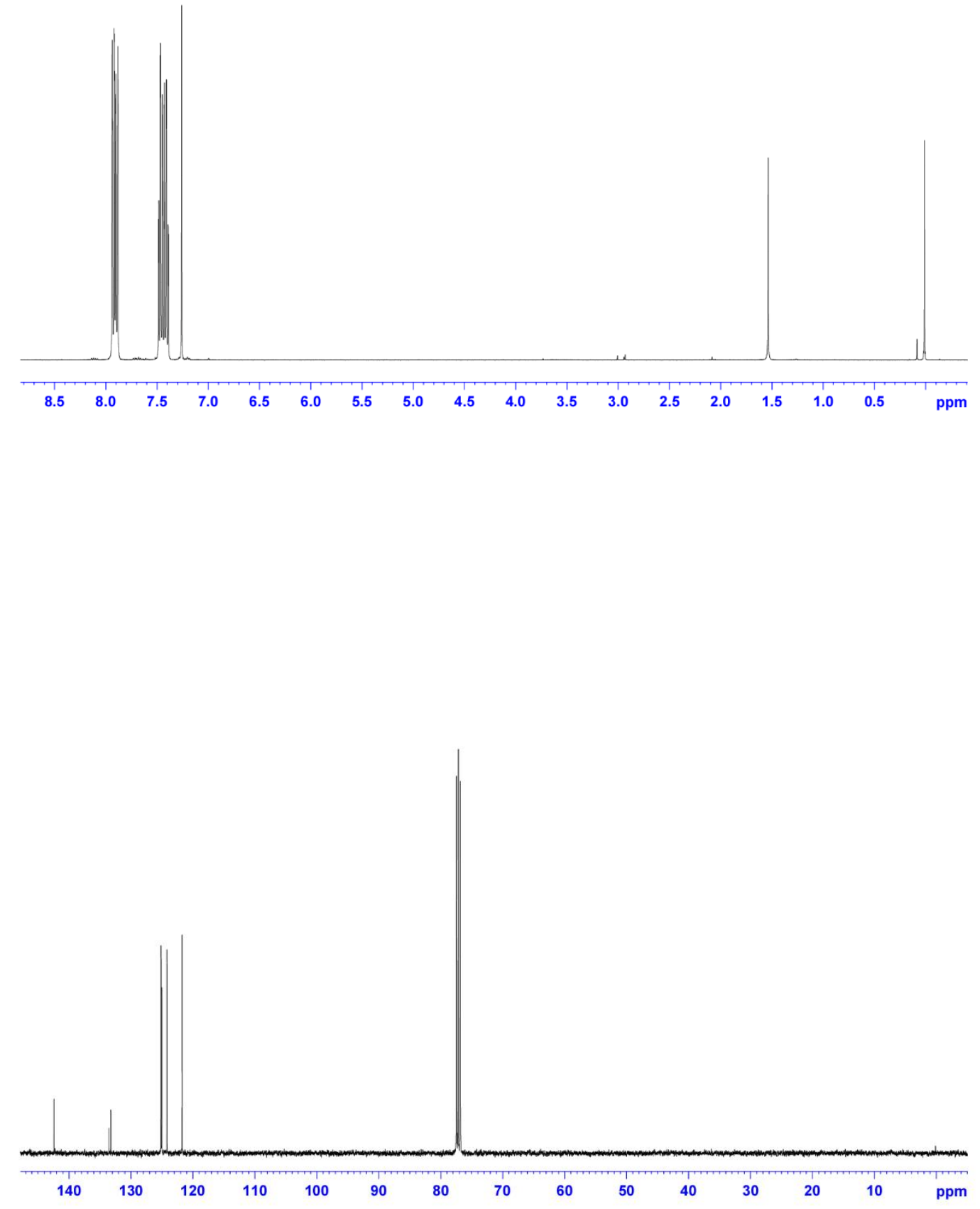
$\left[{ }^{1} \mathrm{H}\right.$ and ${ }^{13} \mathrm{C}$ NMR Spectra of Methyl Ester of $\left.\mathbf{3 b}\right]$<smiles>CC(=O)c1sc2ccccc2c1Sc1cc(C2CCCC2)ccc1Br</smiles>
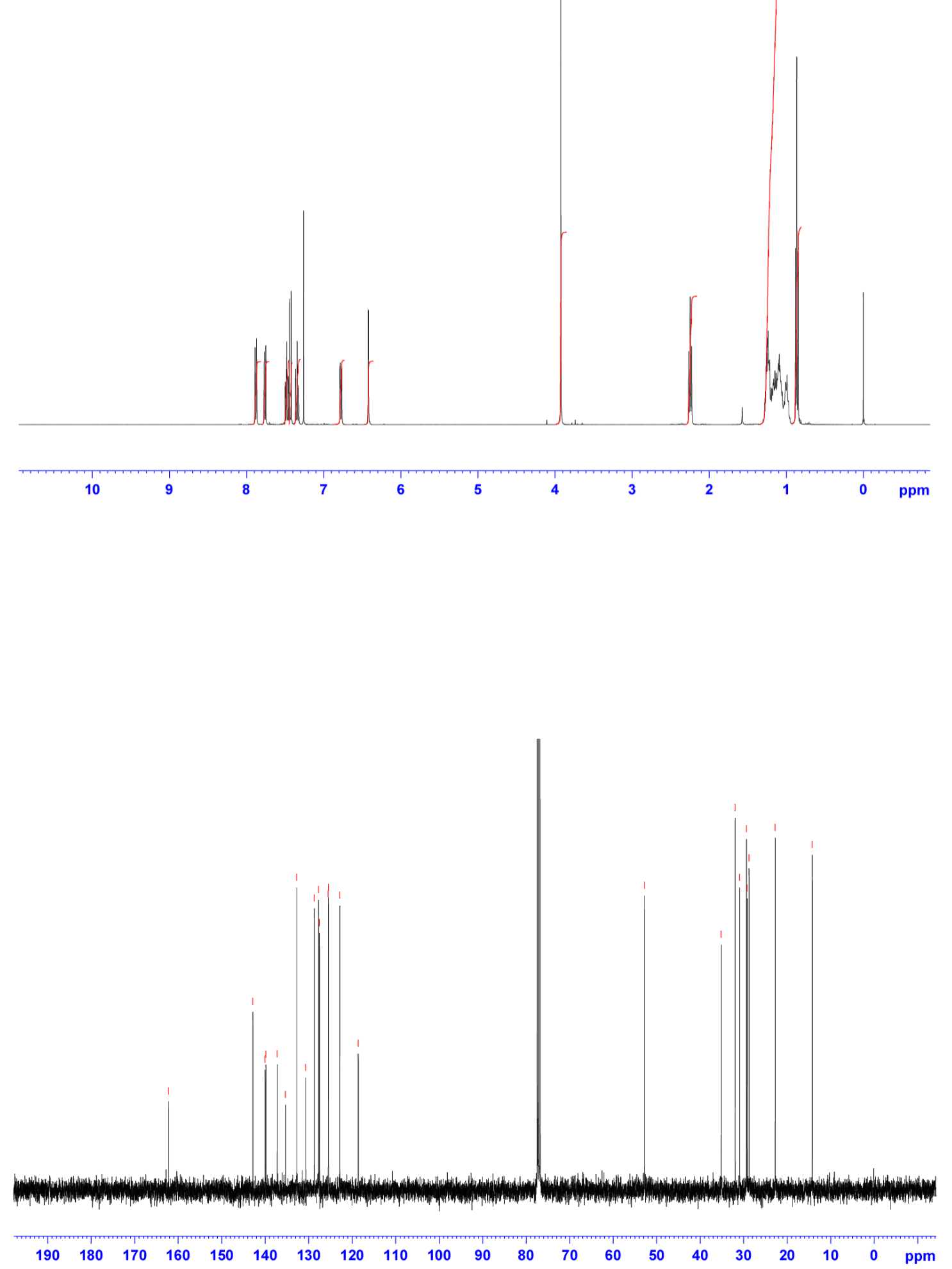
$\left[{ }^{1} \mathrm{H}\right.$ and ${ }^{13} \mathrm{C}$ NMR Spectra of $\left.\mathbf{3 b}\right]$
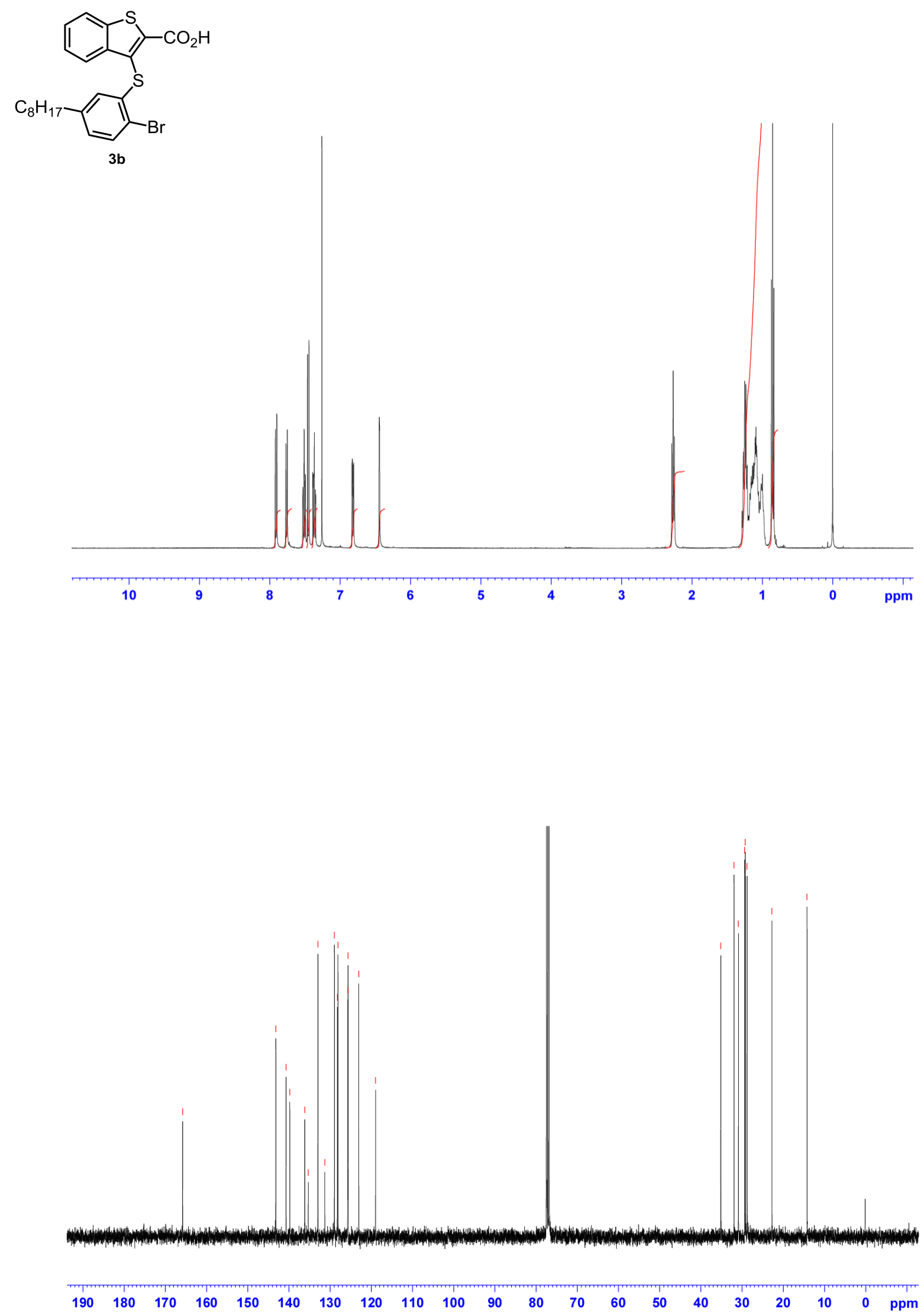
$\left[{ }^{1} \mathrm{H}\right.$ and ${ }^{13} \mathrm{C}$ NMR Spectra of $\left.\mathbf{5 b}\right]$

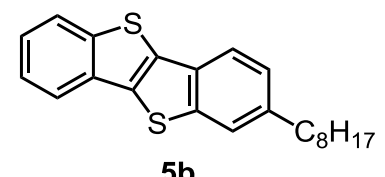

$5 b$
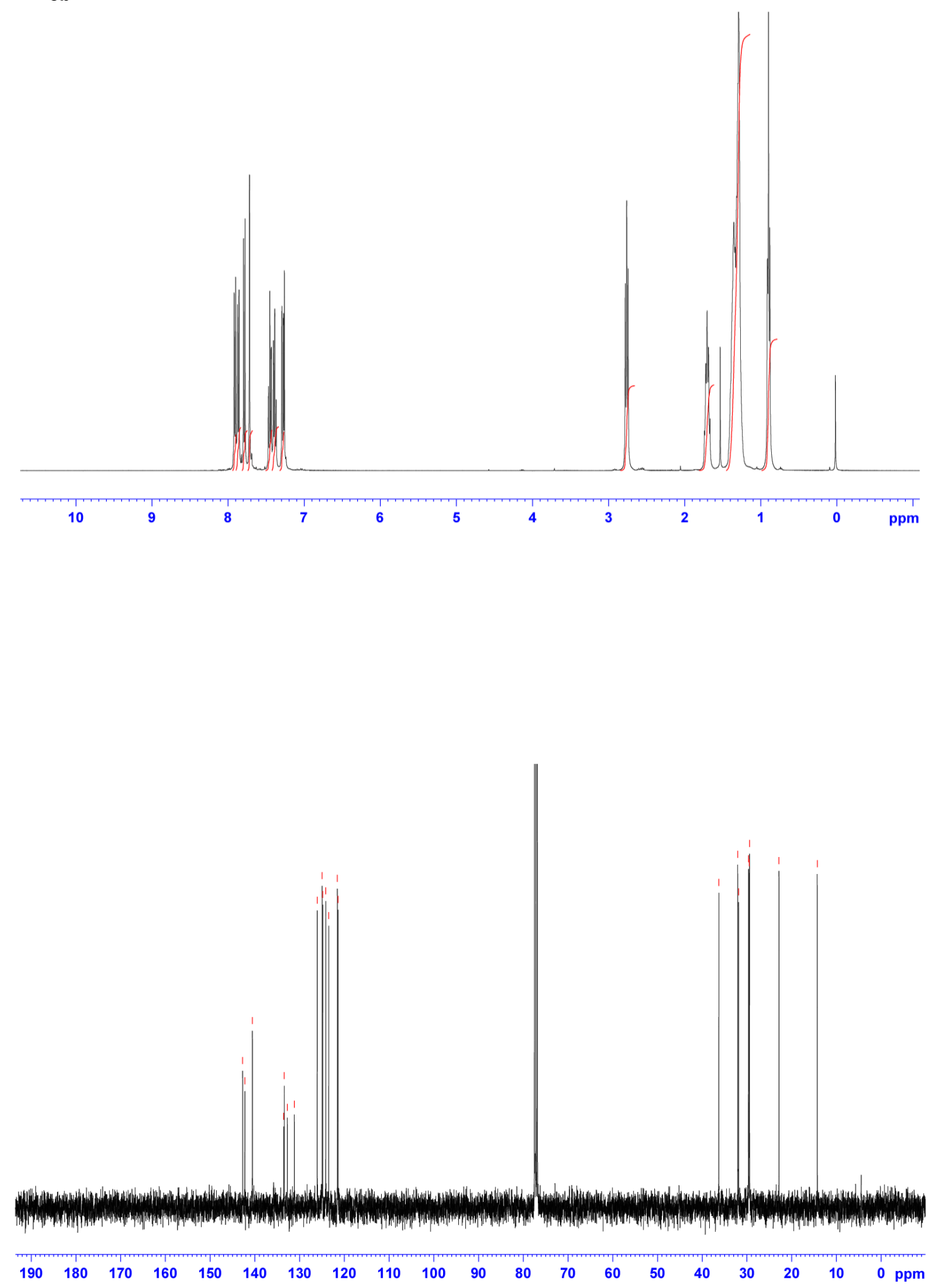
$\left[{ }^{1} \mathrm{H}\right.$ and ${ }^{13} \mathrm{C}$ NMR Spectra of Ethylhexyl Ester of 6a]

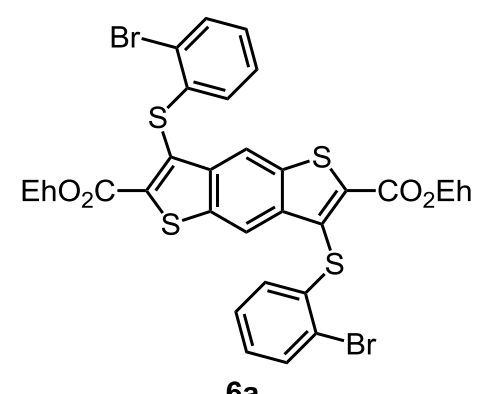

$6 a$
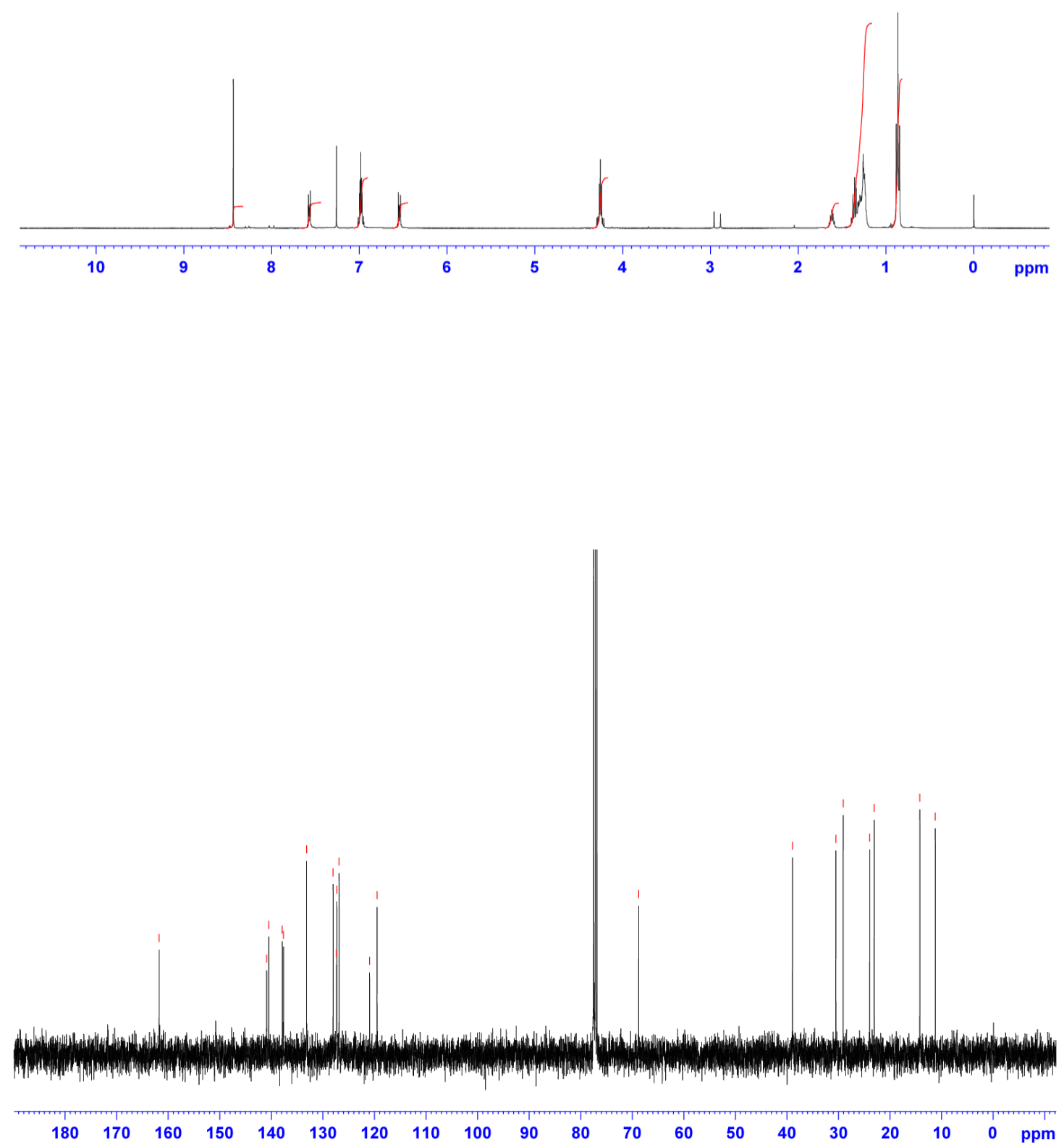
$\left[{ }^{1} \mathrm{H}\right.$ and ${ }^{13} \mathrm{C}$ NMR Spectra of 6a]
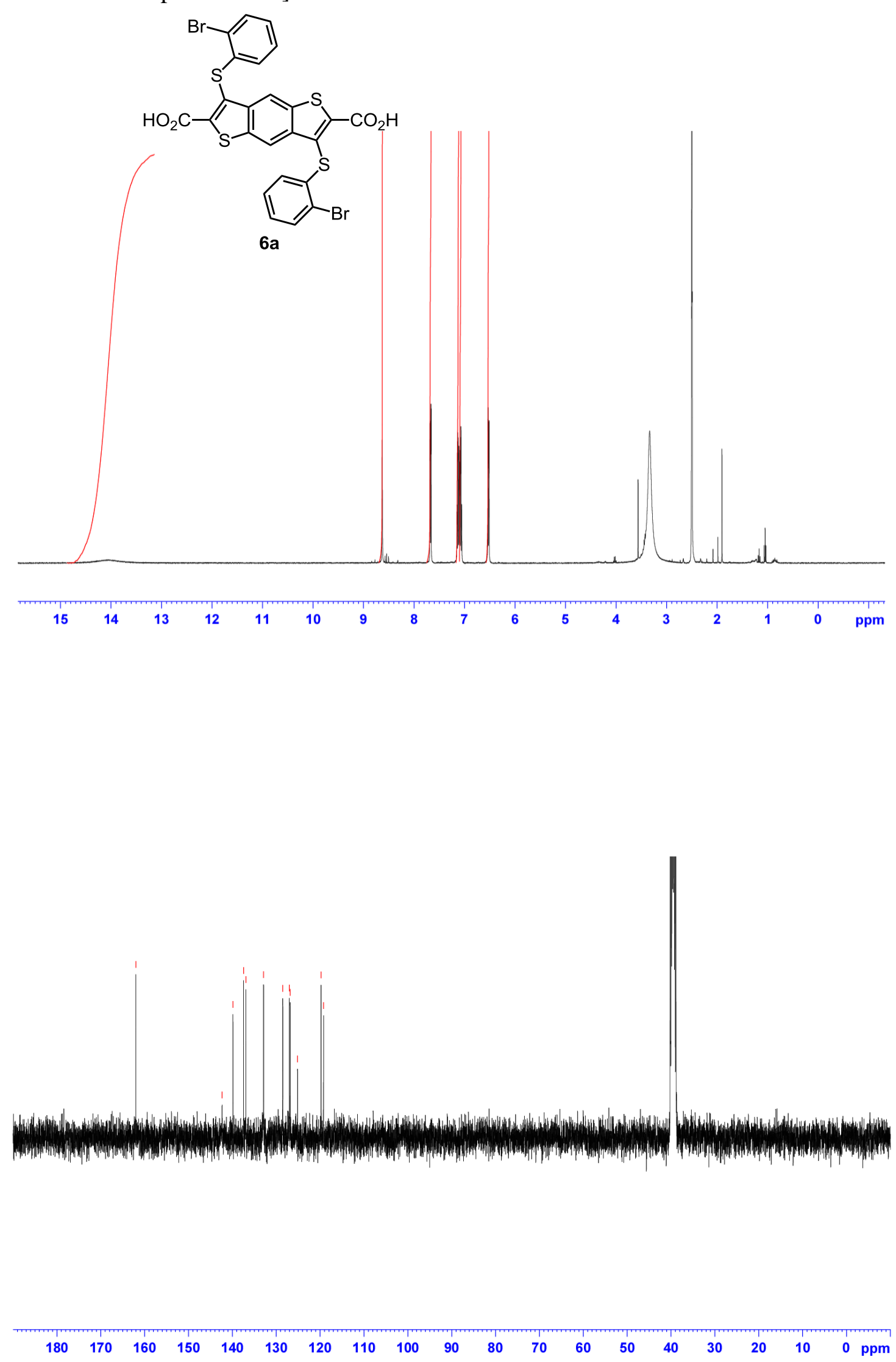

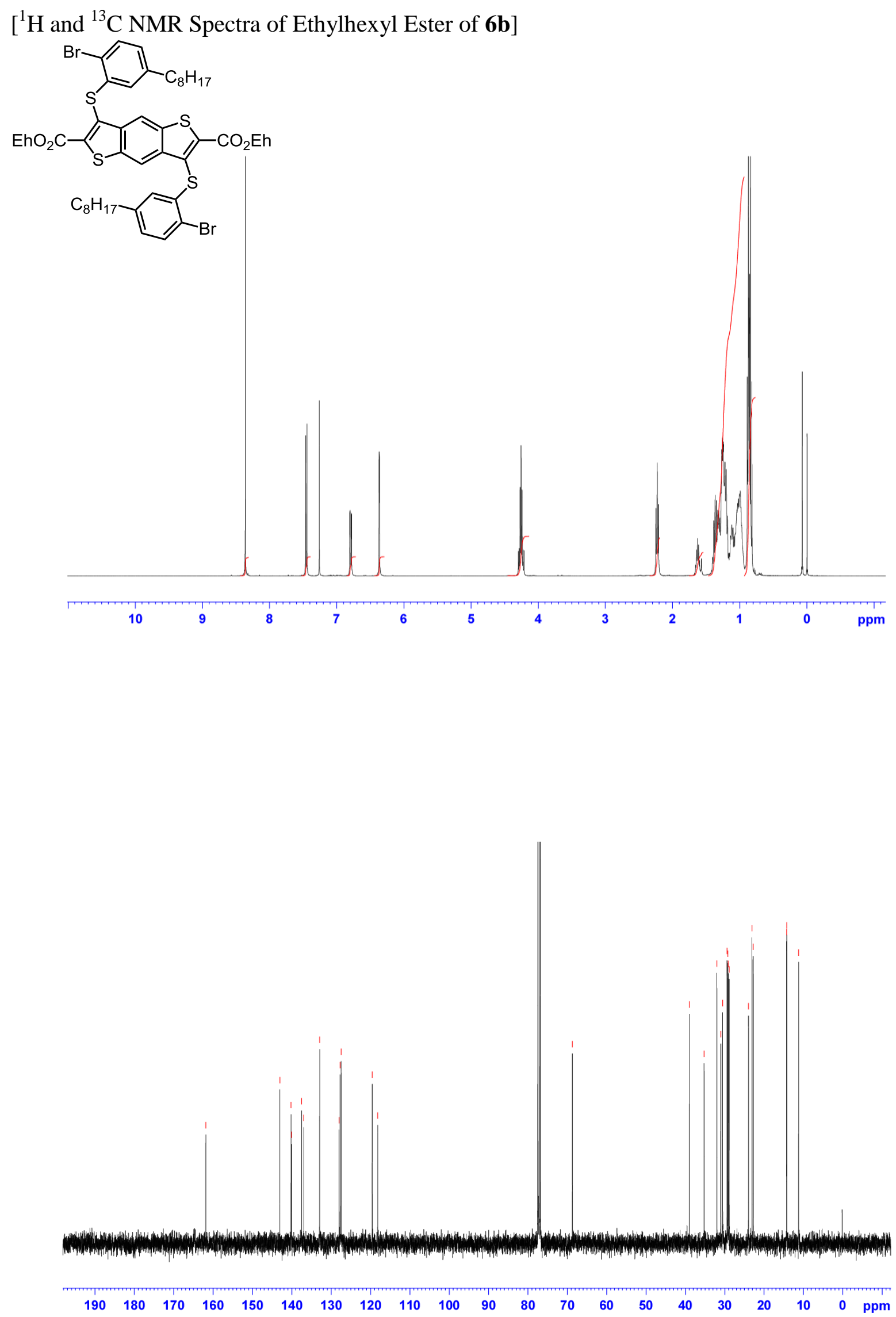
$\left[{ }^{1} \mathrm{H}\right.$ and ${ }^{13} \mathrm{C}$ NMR Spectra of $\left.\mathbf{6 b}\right]$

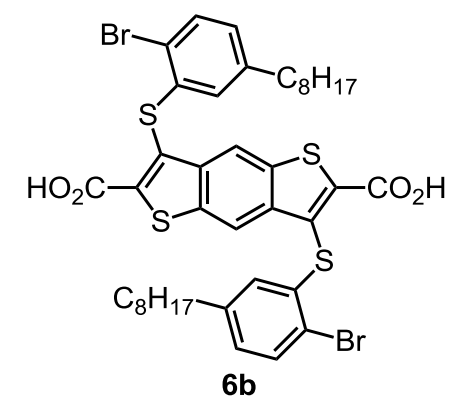

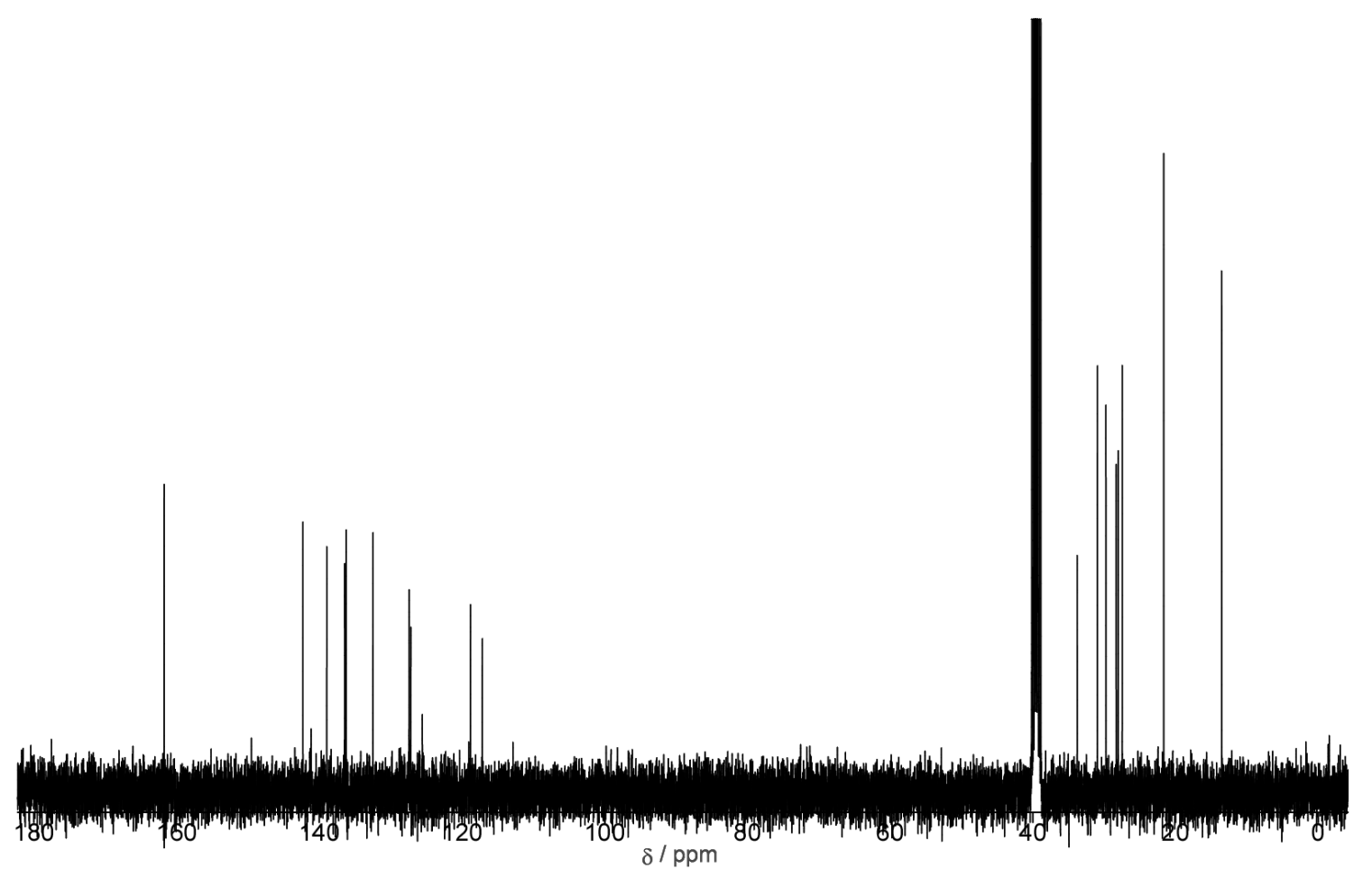


$\left[{ }^{1} \mathrm{H}\right.$ NMR Spectrum of $\left.\mathbf{7 b}\right]$

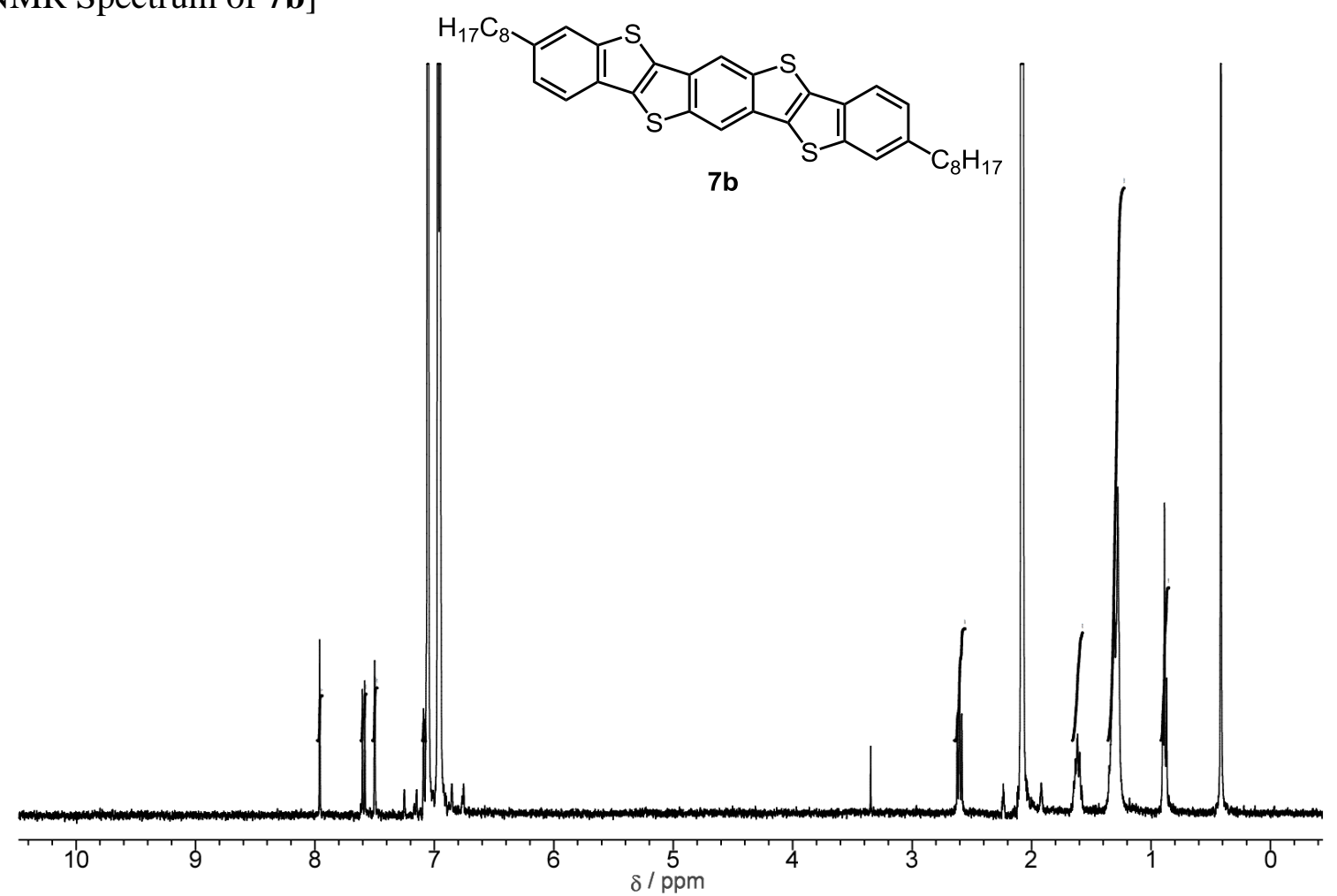


$\left[{ }^{1} \mathrm{H}\right.$ and ${ }^{13} \mathrm{C}$ NMR Spectra of butyl ester of $\left.\mathbf{8 a}\right]$<smiles>O=C(Oc1ccccc1Br)C1=C(Sc2ccccc2)C=Cc2c(sc(C(=O)OCc3ccccc3)c2Sc2ccccc2Br)S1</smiles>

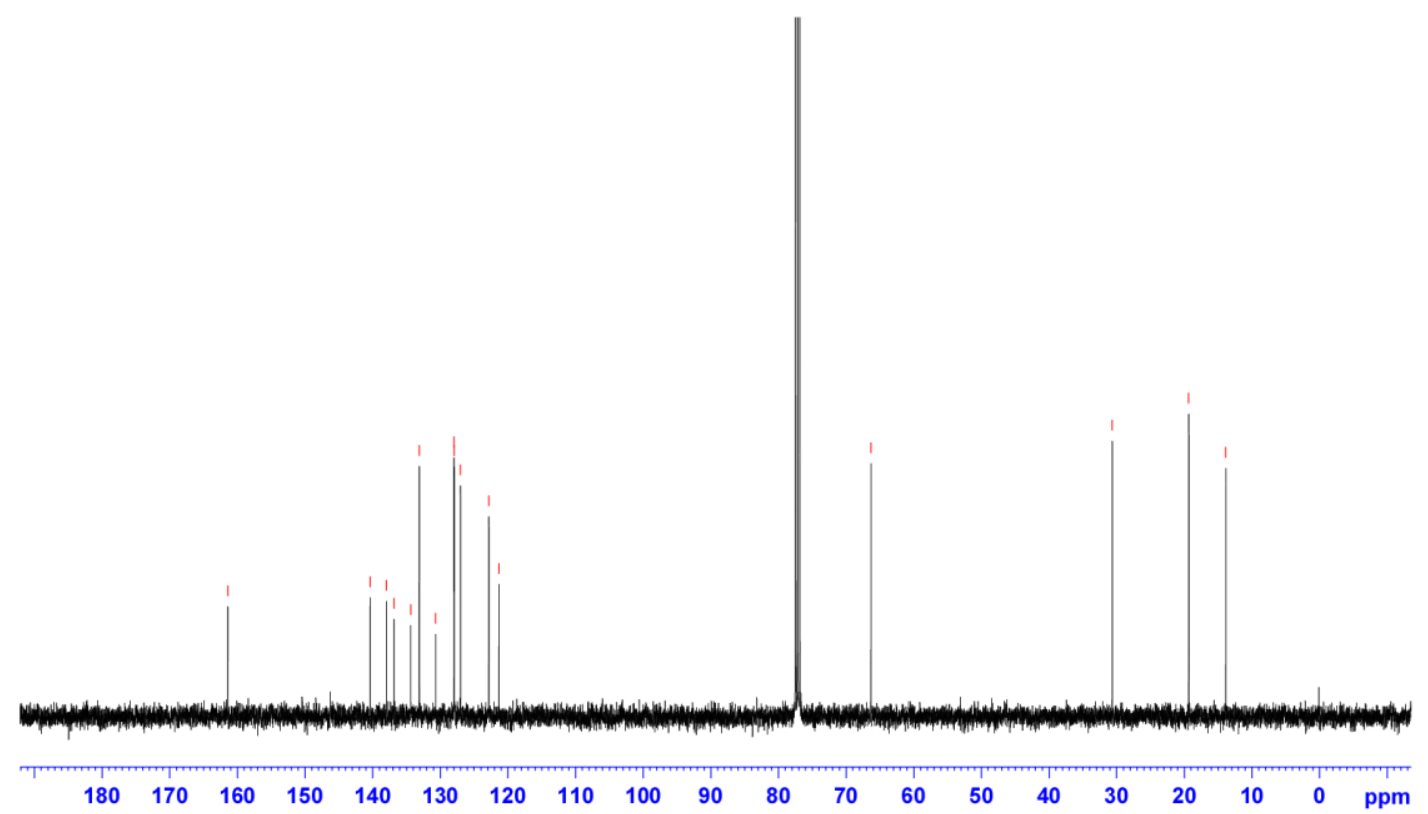


$\left[{ }^{1} \mathrm{H}\right.$ and ${ }^{13} \mathrm{C}$ NMR Spectra of $\left.\mathbf{8 a}\right]$<smiles></smiles>

8a

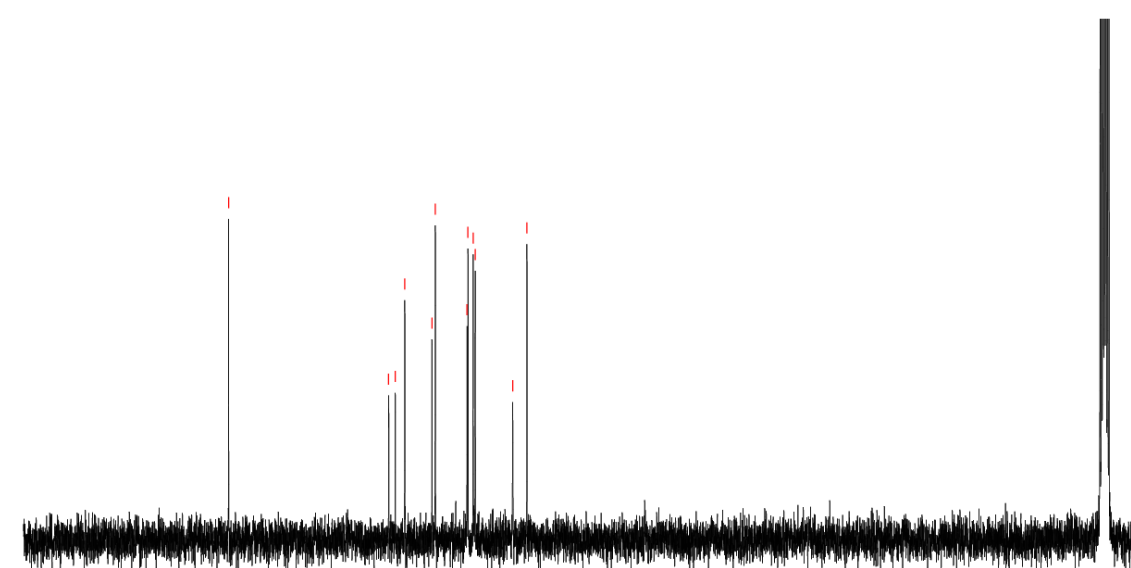

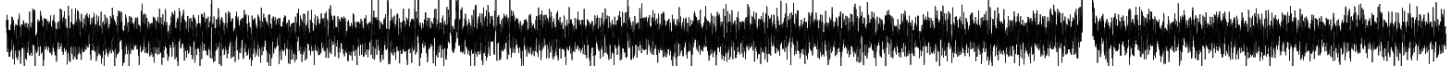

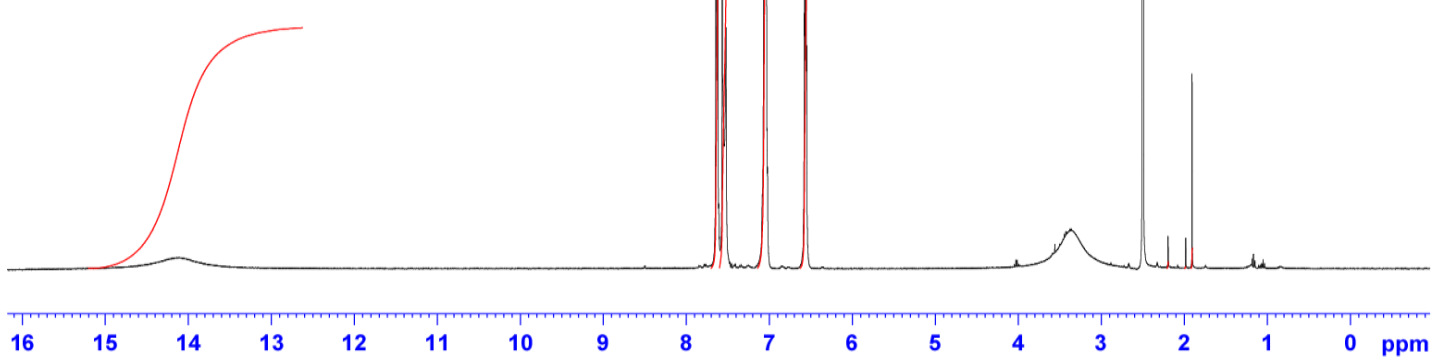

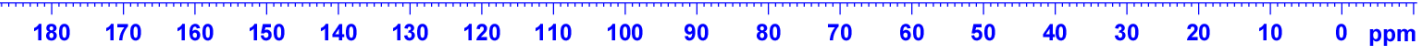


$\left[{ }^{1} \mathrm{H}\right.$ and ${ }^{13} \mathrm{C}$ NMR Spectra of Butyl Ester of $\mathbf{8 b}$ ]

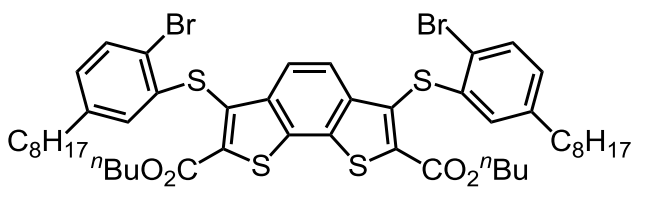
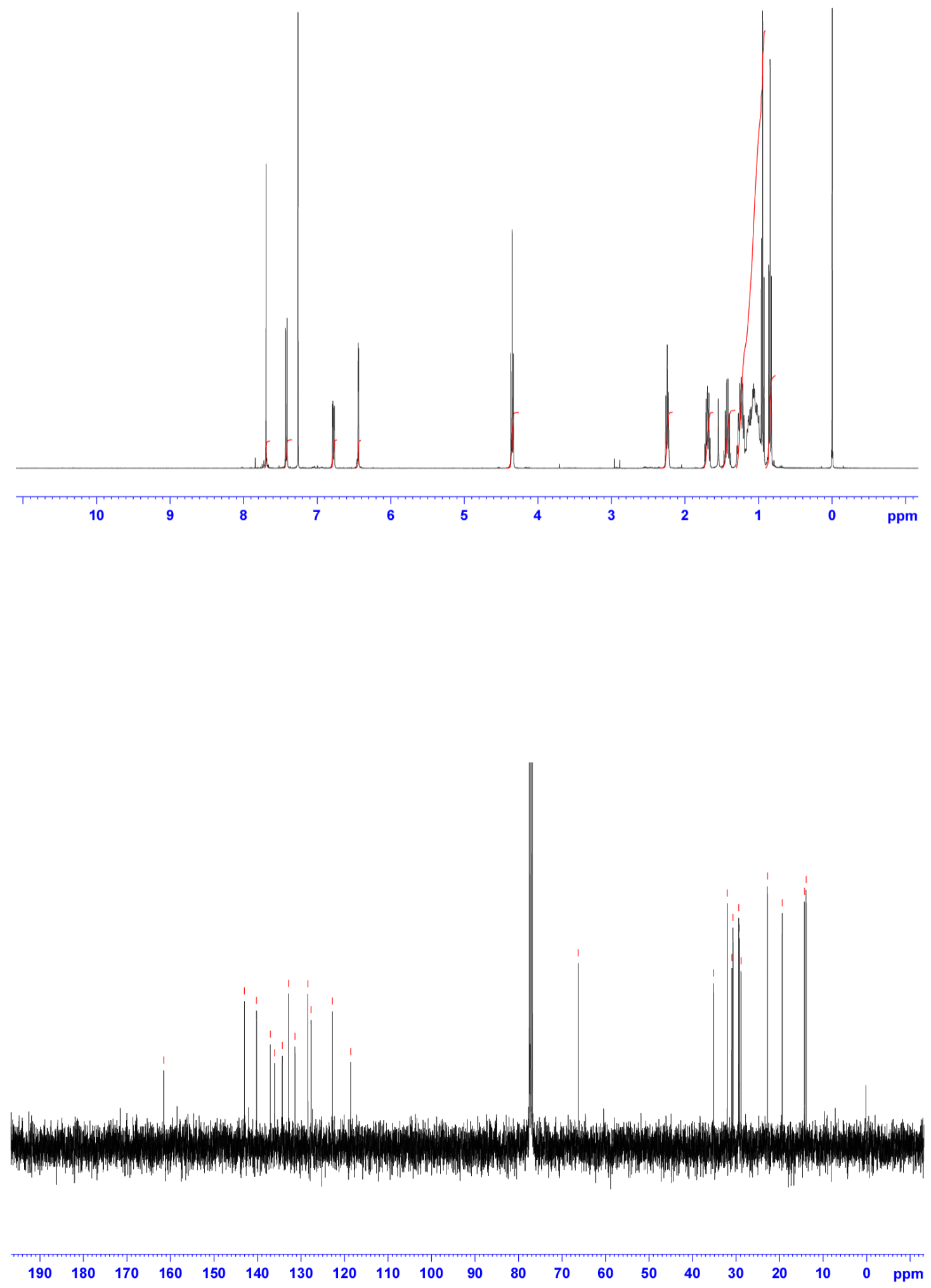
$\left[{ }^{1} \mathrm{H}\right.$ and ${ }^{13} \mathrm{C}$ NMR Spectra of $\left.\mathbf{8 b}\right]$<smiles>CCCCCc1ccc(Br)c(Sc2c(C(=O)O)sc3c2ccc2c(Sc4cc([18F])ccc4Br)c(C(=O)O)sc23)c1</smiles>

$8 b$

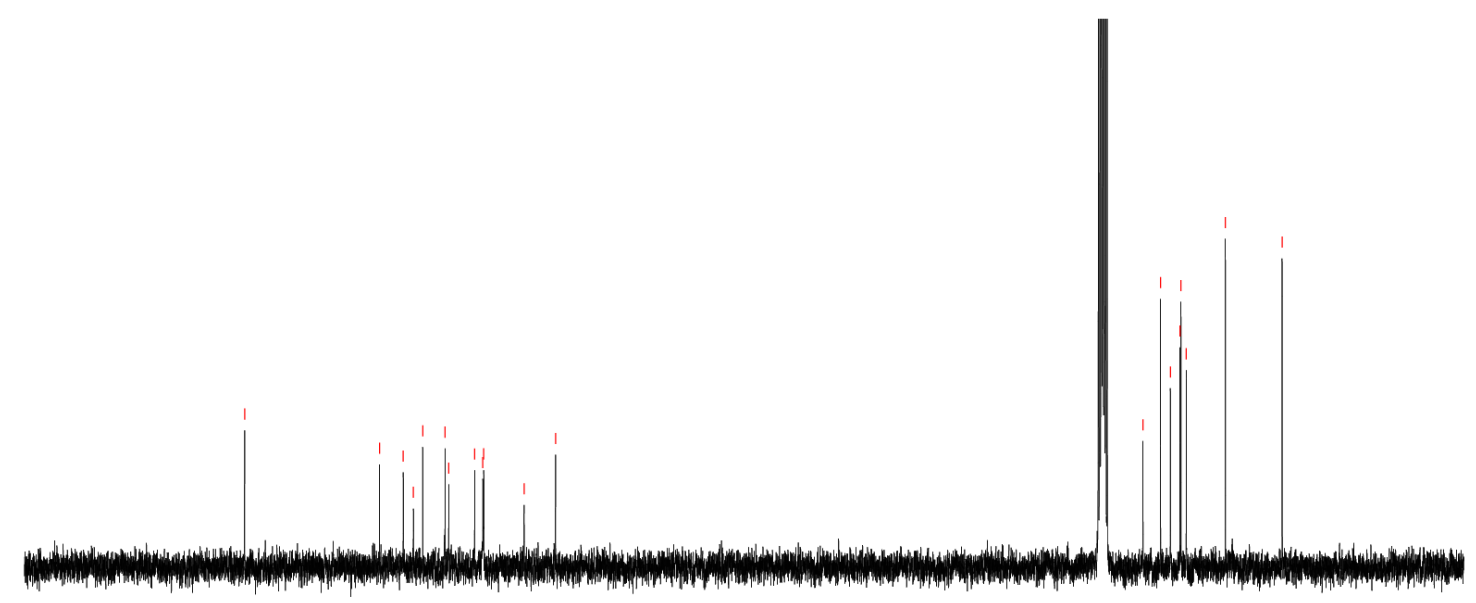

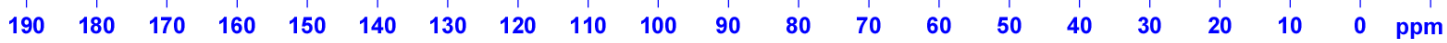


$\left[{ }^{1} \mathrm{H}\right.$ NMR Spectrum of 9b]

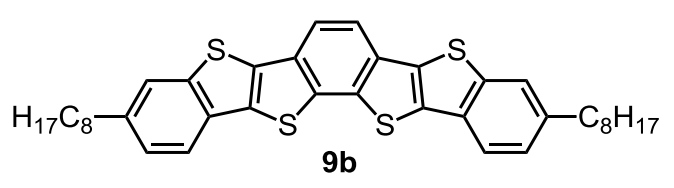

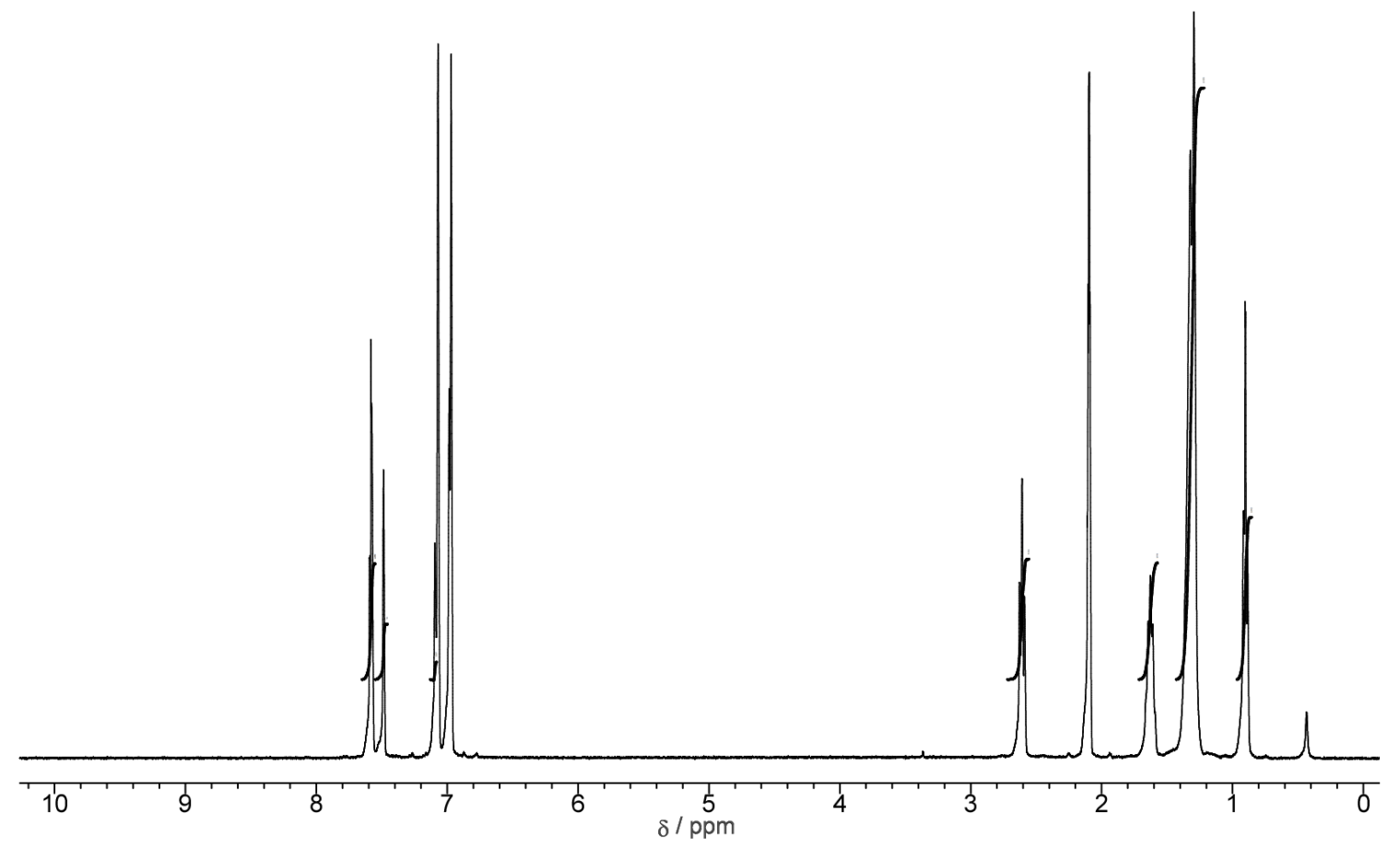

